\title{
Scaling Laws for Ergodic Spectral Efficiency in MIMO Poisson Networks
}

\author{
Junse Lee, Namyoon Lee and François Baccelli
}

\begin{abstract}
In this paper, we examine the benefits of multiple antenna communication in random wireless networks, the topology of which is modeled by stochastic geometry. The setting is that of the Poisson bipolar model introduced in [1], which is a natural model for ad-hoc and device-to-device (D2D) networks. The primary finding is that, with knowledge of channel state information between a receiver and its associated transmitter, by zero-forcing successive interference cancellation, and for appropriate antenna configurations, the ergodic spectral efficiency can be made to scale linearly with both 1) the minimum of the number of transmit and receive antennas, 2) the density of nodes and 3) the path-loss exponent. This linear gain is achieved by using the transmit antennas to send multiple data streams (e.g. through an open-loop transmission method) and by exploiting the receive antennas to cancel interference. Furthermore, when a receiver is able to learn channel state information from a certain number of near interferers, higher scaling gains can be achieved when using a successive interference cancellation method. A major implication of the derived scaling laws is that spatial multiplexing transmission methods are essential for obtaining better and eventually optimal scaling laws in multiple antenna random wireless networks. Simulation results support this analysis.
\end{abstract}

\section{INTRODUCTION}

A multiple-input-multiple-output mobile ad hoc network (MIMO-MANET) is an infrastructureless network in which a large number of transmit-and-receive pairs, each with multiple antennas, communicate by sharing some common spectrum [2], [3]. Such networks are fundamental in a

J. Lee and F. Baccelli are with the Wireless Networking and Communications Group, Department of Electrical and Computer Engineering, The University of Texas at Austin, Austin, TX 78712 USA (e-mali: junselee@utexas.edu and francois.baccelli@austin.utexas.edu) N. Lee is with the Department of Electrical Engineering, POSTECH, Pohang, Gyeongbuk, Korea 37673 (e-mail:nylee@ postech.ac.kr). 
variety of applications including car-to-car and device-to-device communication systems [4]-[6]. It is therefore of great importance to characterize the system-level performance of such networks [7]-[9].

Despite extensive research over a few decades, analytical expressions for the spectral efficiency of such systems are still missing. The principal difficulty has been the lack of a tractable model quantifying uncoordinated inter-node interference together with inter-stream interference at a receiver equipped with multiple antennas. In this paper, we leverage two analytical tools to cope with this difficulty. The first one is stochastic geometry which models the locations of links as Poisson dipoles [1] and allows one to compute the distribution of the interference power. The second one is random matrix theory [10], which is exploited for calculating the distribution of inter-stream interference power under different MIMO detection techniques. Combining these tools, we characterize the ergodic spectral efficiencies and the scaling laws of a super-dense MIMO-MANET system, under Poisson assumptions on the node locations, and when considering two major types of channel knowledge at receivers. By leveraging the closed-form expressions which are derived, we highlight the interplay among four key system parameters determining the scaling laws, namely the number of antennas at the transmitter, the number of antennas at the receiver, the node density, and the path-loss exponent.

\section{A. Related Works}

There has been extensive work on the capacity of MIMO-MANETs. MIMO-MANETs can be modeled as MIMO interference networks in which a finite number of transmit-and-receiver pairs communicate by sharing the same spectrum, without transmitter cooperation. [7] studied the capacity of a MIMO-MANET by treating inter-node interference as additional noise at a receiver, and derived the optimal power allocation strategy for the MIMO transmission. For instance, in a certain range of interference-to-noise ratios, it turns out that allocating the whole power to one antenna (i.e., using a single stream transmission) is optimal. [8] and [11] extended the result of [7], and demonstrated that the asymptotic spectral efficiency is improved by sending multiple data streams. A common assumption of these studies is that the distances between any two nodes in the network are deterministic [7] or identical [8], which is unrealistic to model MANETs in practice. This approach cannot be used to assess which MIMO transmission techniques provide the highest gains in large random MANETs. 
When considering more realistic random network topology assumptions, the rates achievable in MANETs have been studied in [2], [9], [12]-[16]. The study of scaling laws within this context was initiated by Gupta and Kumar's seminal paper [2]. Under the assumption that $n$ nodes are randomly located in the unit disk, Gupta and Kumar showed that multihop routing based on a decode-and-forward scheme can reach to a total throughput which scales as $\mathcal{O}(\sqrt{n})$. By using percolation theory, it was later shown in [12] that a better scaling law of order $\mathcal{O}(\sqrt{n / \log n})$ is achievable. Subsequently, improved scaling results were derived in MANETs, assuming that some specific additional assumptions hold on mobility [14], bandwidth [15], or node-cooperation [9]. The main differences between our work and this line of research are the following: (1) our model is based on Poisson dipoles and assumes that source-destination pairs communicate with each other relying upon single-hop transmissions, i.e., neither multi-hop routing schemes nor node-cooperation are allowed (in a sense, the present paper is more focussed on D2D than on MANETs). (2) we focus on the use of multiple antennas at both transmitters and receivers, while this line of research was centered on the scenario with a single antenna at both transmitters and receivers. (3) our performance metric is spatially-averaged ergodic spectral efficiency, while the work alluded to above focused on transport capacity. (4) even if new scaling laws are our main results, our approach also provides exact formulas for the mean Shannon rate of a typical link and the spectral efficiency per unit area (see e.g. Theorems b1 and 2 below), and goes hence beyond the scaling law setting.

In the present paper, we assume that the interferer locations are Poisson distributed over the plane [17], [18], which is an appropriate model for e.g. D2D, where transmitters are randomly located in an uncoordinated manner. Using this model, the transmission capacity of ad hoc networks, which quantifies the maximum allowable spatial density of successful transmissions per unit area, subject to a given outage probability constraint, was characterized in certain settings. For example, the transmission capacity expressions of ad hoc networks were found when adopting spread spectrum techniques [19], [20], interference cancellation [21]-[23], and multiple-antenna transmission methods [24]-[31]. In particular, in [25], it was demonstrated that interference cancellation techniques at a receiver employing multiple antennas can provide a linear increase of the transmission capacity of ad hoc networks with the node density. In [29], it was shown that for a MIMO setting, a single stream transmission is optimal in terms of transmission capacity, when all the degrees of freedom of the receive antennas are used for interference cancellation. 
Arguably, a common shortcoming of the transmission capacity metric is that it cannot capture the effects of rate adaptation techniques, which are the key features used in many modern wireless systems to track and exploit channel variations [32]. The main novelty of the present paper compared to this line of thought is the analysis of the ergodic spectral efficiency (rather than transport capacity), which quantifies the achievable Shannon transmission rate per unit area when adapting the rate to the different local conditions. For a single-input-multiple-output (SIMO) setting, the recent work in [33] showed that the sum spectral efficiency per link can increase linearly with both the density and the path loss exponent provided the number of antennas is a linear function of the density. For a MIMO setting, however, it is still unknown whether spatial multiplexing transmission techniques [34] can improve the scaling laws of the sum spectral efficiency. We recall that spatial multiplexing consists in transmitting different data streams on the transmit antennas and in identifying/discriminating between these streams at the receiver, while transmit diversity consists in sending the same data symbols over multiple transmit antennas to enhance the reliability. The main qualitative achievement of this paper is a proof that the answer to this question is positive and more precisely the identification of the network densities and antenna configurations for which spatial multiplexing strategies achieve higher sum spectral efficiency per unit area than the methods based on transmit diversity.

\section{B. Main Contributions}

We consider a random network the topology of which modeled by a Poisson bipolar network [1] with density $\lambda$ on $\mathbb{R}^{2}$. In this model, each transmitter has its receiver at some random distance. Each transmitter is equipped with $N_{\mathrm{t}}$ antennas and is assumed to send $N_{\mathrm{t}}$ data streams to its associated receiver, equipped with $N_{\mathrm{r}}\left(\geq N_{\mathrm{t}}\right)$ antennas. Our key findings can be summarized as follows:

- We first consider the case where each receiver has knowledge of the state of the channel between its transmitter and itself only. We refer to this channel knowledge assumption as direct channel state information (DCSI) at receiver (DCSIR). Under the premise of this channel knowledge, and under zero-forcing (ZF) detection and ZF-based successive interference cancellation (ZF-SIC) detection respectively, we derive analytical expressions of the sum spectral efficiency as a function of 1) the network density $\lambda, 2)$ the number of transmit and receive antennas $\left(N_{\mathrm{t}}\right.$ and $\left.\left.N_{\mathrm{r}}\right), 3\right)$ the path-loss exponent $\alpha$, and 4) the 
signal-to-noise ratio (SNR). By deriving a closed form lower and upper bound on this sum spectral efficiency, we show that, as $\lambda$ goes to infinity, when $N_{\mathrm{t}}=c_{1} \lambda^{\beta_{1}}, N_{\mathrm{r}}=c_{2} \lambda^{\beta_{2}}$ for some constants $c_{1}, c_{2}>0, \beta_{1} \leq \beta_{2}$ and $\alpha>2$, the scaling laws of the ergodic spectral efficiency per link is

$$
\Theta\left(\lambda^{\beta_{1}} \log _{2}\left(1+\lambda^{\beta_{2}-\beta_{1}-\frac{\alpha}{2}}\right)\right)
$$

for both ZF and ZF-SIC. One important implication of this scaling law is that when $\beta_{2} \geq$ $\beta_{1}+\frac{\alpha}{2}$, transmitting multiple streams is more beneficial in the scaling law sense than sending a single stream, which strongly contrasts with the result derived on transmission capacity [29]. In fact, this result agrees with the intuition that it should be possible to improve the data rates per link by having $N_{\mathrm{t}}=c_{1} \lambda^{\beta_{1}}$ and by transmitting multiple data streams (multiplexing gain), provided the remaining degrees of freedom at the receiver are sufficient to cancel both inter-stream interference and inter-node interference and to discriminate between the independent data streams. Furthermore, this scaling law expression generalizes the result for the SIMO case derived in [33] to the MIMO case.

- We also consider the case where each receiver is able to learn the CSI of its $L$-nearest interferers with $\left(0<L \leq\left\lfloor\frac{N_{\mathrm{r}}}{N_{\mathrm{t}}}\right\rfloor-1\right) 1$, which is referred to here as local CSIR (LCSIR). Using a ZF-SIC detection technique for suppressing both inter-stream and inter-node interference, we give an exact expression of the sum spectral efficiency. By leveraging this expression, we get an achievable scaling law of the sum spectral efficiency per link of the form:

$$
\Omega\left(\lambda^{\beta_{1}}\left(\log _{2}\left(1+\lambda^{\left(\beta_{2}-\beta_{1}-1\right) \frac{\alpha}{2}}\right)\right)\right)
$$

when $N_{\mathrm{t}}=c_{1} \lambda^{\beta_{1}}$ and $N_{\mathrm{r}}=c_{2} \lambda^{\beta_{2}}$, for some constants $c_{1}, c_{2}>0$ and $\beta_{1} \leq \beta_{2}, \alpha>2$, and for $L=\left\lfloor\frac{N_{\mathrm{r}}}{N_{\mathrm{t}}}\right\rfloor-1$. This result also demonstrates that the MIMO transmission method improves the scaling law of the ergodic spectral efficiency per link by increasing multiplexing gains, provided $\beta_{2} \geq \beta_{1}+1$. Comparing to DCSIR, with LCSIR, it is possible to increase the sum spectral efficiency with both the path-loss exponent and the number of transmit antennas. This multiplicative gain in the achievable scaling law comes from the fact that the receiver exploits LCSIR.

\footnotetext{
${ }^{1}\lfloor x\rfloor$ denotes the largest integer no more than $x$.
} 
This paper is organized as follows. The network model, the performance metrics, and the receiver schemes are discussed in Section III. The exact expression and the scaling law for ergodic spectral efficiency are provided in Section III in the DCSIR case and in Section IV] in the LCSIR case. We conclude in Section $\mathrm{V}$.

\section{SySTEM MODEL}

\section{A. Network Model}

We consider a Poisson bipolar network model which features an infinite number of transmitterreceiver pairs scattered in the Euclidean plane. Let $\Phi=\left\{X_{i}\right\}_{i \in \mathbb{N}}$ denote the locations of the transmitters, which are assumed to form some realization of a homogeneous PPP with positive and finite intensity $\lambda$ on $\mathbb{R}^{2}$. Let $\bar{\Phi}=\left\{Y_{i}\right\}_{i \in \mathbb{N}}$ denote the locations of the receivers. The receiver $Y_{i}$ of $X_{i}$ is assumed to be uniformly distributed on a ring with inner radius 1 and outer radius $R_{d}$ centered at $\left\{X_{i}\right\}$, where $R_{d}>1$. Fig. 1 provides a snapshot of network topology with $R_{d}=50 \mathrm{~m}$ and $\lambda=0.000004 / \mathrm{m}^{2}$. We assume that each receiver is equipped with $N_{\mathrm{r}}$ antennas, whereas transmitters have a random number $N_{\mathrm{t}}$ in $\left[1, N_{\mathrm{r}}\right] \in \mathbb{N}$ of antennas. We denote the probability of having $k$ transmit antennas by $p_{k}$ where $\sum_{k=1}^{N_{\mathrm{r}}} p_{k}=1$. These numbers of transmit are assumed independent and identically distributed (i.i.d.) over links.

\section{B. Signal Model}

A transmitter $X_{k} \in \Phi$ communicates with its associated receiver $Y_{k}$, and sends a signal $\mathbf{s}_{k} \in \mathbb{C}^{N_{\mathrm{t}, k} \times 1}$ when $X_{k}$ has $N_{\mathrm{t}, k}$ antennas, with power constraint $\mathbb{E}\left[\left\|\mathbf{s}_{k}\right\|^{2}\right]=P$. Here, we assume the transmit power is equally allocated to all antennas. Assuming a frequency-flat channel, the received signal at the $k$-th receiver, $\mathbf{y}_{k} \in \mathbb{C}^{N_{\mathrm{r}} \times 1}$ is

$$
\mathbf{y}_{k}=\sum_{l, X_{l} \in \Phi} d_{k, l}^{-\frac{\alpha}{2}} \mathbf{H}_{k, l} \mathbf{s}_{l}+\mathbf{z}_{k}
$$

where $\mathbf{H}_{k, l} \in \mathbb{C}^{N_{\mathrm{r}} \times N_{\mathrm{t}, l}}$ is the channel matrix and $d_{k, l}$ the distance from $X_{l}$ to $Y_{k}$, respectively. Moreover, $\mathbf{z}_{k} \in \mathbb{C}^{N_{\mathrm{r}} \times 1}$ is the noise vector at receiver $Y_{k}$. Furthermore, we assume that all entries of $\mathbf{H}_{k, l}$ are i.i.d. complex Gaussian random variables with zero mean and unit variance, i.e. $\mathcal{C N}(0,1)$, and that all entries of $\mathbf{z}_{k}$ are i.i.d. $\mathcal{C N}\left(0, \sigma^{2}\right)$, where $\sigma^{2}$ is the noise variance. 


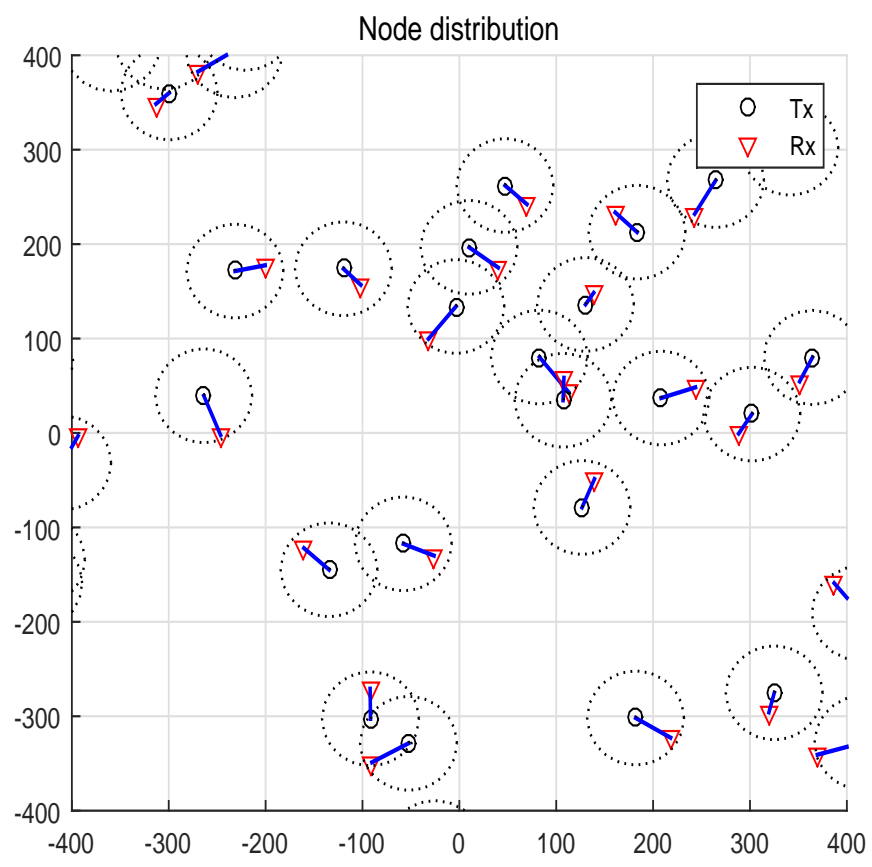

Fig. 1: A snapshot of bipolar MANET where $\lambda=0.00004 / \mathrm{m}^{2}$ and $R_{d}=50 \mathrm{~m}$.

\section{Receive Filters and Performance Metrics}

We assume that receiver $Y_{k}$ can measure CSI from its associated transmitter $X_{k}$ and from the $L_{k}$ nearest transmitters, i.e. $\left\{X_{k_{i}}\right\}_{i=1}^{L_{k}}$, where $0 \leq L_{k} \leq \max \left\{n \mid \sum_{i=1}^{n} N_{\mathrm{t}, j_{i}} \leq N_{\mathrm{r}}-N_{\mathrm{t}, k}\right\}$. It will be assumed that $X_{k}$ sends $N_{\mathrm{t}, k}$ data streams without using any precoding, i.e., that an open-loop MIMO transmission is used, and also that the receiver uses linear receive filters to detect the desired data symbol to eliminate the inter-stream interference and the inter-node interference.

Let $\mathbf{v}_{k}(m) \in \mathbb{C}^{N_{\mathrm{r}} \times 1}, m=1, \ldots, N_{\mathrm{t}, k}$, denote the receive filter vector used at $Y_{k}$ for detecting the $m$-th data stream of its transmitter. Then, the resulting signal-to-interference-and-noise ratio (SINR) for the $m$-th data stream of the $k$-th link is

$$
\operatorname{SINR}_{k}(m)=\frac{H_{k, k}(m) d_{k, k}^{-\alpha}}{I_{k 1}(m)+I_{k 2}(m)+I_{k 3}(m)+\frac{N_{\mathrm{t}, k} \sigma^{2}}{P}},
$$

\footnotetext{
${ }^{2}$ With this condition, the number of received data streams at $Y_{k}$ is no larger than $N_{\mathrm{r}}$. This assumption is necessary for decoding the independent data streams in ZF and ZF-SIC. If all transmitters are equipped with $N_{\mathrm{t}}$ antennas, $L_{k}=\left\lfloor\frac{N_{\mathrm{r}}}{N_{\mathrm{t}}}\right\rfloor-1, \forall k \in \mathcal{K}$. Further, we denote the $j$-th nearest interferer from $Y_{k}$ by $X_{k_{j}}$.
} 
where

$$
\begin{array}{cc}
H_{k, k}(m)=\left\|\mathbf{v}_{k}^{*}(m) \mathbf{H}_{k, k}(:, m)\right\|^{2}, & I_{k 1}(m)=\sum_{i=1, \ldots, N_{\mathrm{t}, k}}^{i \neq m}\left\|\mathbf{v}_{k}^{*}(m) \mathbf{H}_{k, k}(:, i)\right\|^{2} d_{k, k}^{-\alpha}, \\
I_{k 2}(m)=\sum_{l \in\left\{k_{1}, k_{2}, \ldots, k_{L_{k}}\right\}}\left\|\mathbf{v}_{k}^{*}(m) \mathbf{H}_{k, l}\right\|^{2} d_{k, l}^{-\alpha}, \quad I_{k 3}(m)=\sum_{l, X_{l} \in\left\{\Phi \backslash X_{k}, X_{k_{1}}, X_{k_{2}}, \ldots, X_{k_{L_{k}}}\right\}}\left\|\mathbf{v}_{k}^{*}(m) \mathbf{H}_{k, l}\right\|^{2} d_{k, l}^{-\alpha} .
\end{array}
$$

Here, we denote the conjugate transpose by $*$ and the $i$-th column of the matrix $A$ by $A(:, i)$. As can be seen in (4), the total amount of interference at the receiver can be decomposed into three factors: 1) the inter-stream interference $I_{k 1}, 2$ ) the inter-node interference from the $L_{k}$-dominant interferers, $I_{k 2}$, and 3) the inter-node interference, $I_{k 3}$, which is the interference from the other nodes. Then, the achievable rate of the $k$-th link is

$$
C_{k}=\sum_{m=1}^{N_{\mathrm{t}, k}} C_{k}(m)=\sum_{m=1}^{N_{\mathrm{t}, k}} \log _{2}\left(1+\operatorname{SINR}_{k}(m)\right) .
$$

The main target performance metric in this paper is ergodic spectral efficiency. The sum spectral efficiency per unit area is defined by

$$
C=\frac{1}{|\mathcal{A}|} C_{\mathcal{A}}=\frac{1}{|\mathcal{A}|} \mathbb{E}\left[\sum_{k, X_{k} \in \mathcal{A}} C_{k}\right]=\lambda \mathbb{E}^{0}\left[\sum_{m=1}^{N_{\mathrm{t}, 0}} \log _{2}\left(1+\operatorname{SINR}_{0}(m)\right)\right],
$$

where for any $\mathcal{A} \subset \mathbb{R}^{2},|\mathcal{A}|$ is the area of $\mathcal{A}, C_{\mathcal{A}}$ is the sum spectral efficiency of $\mathcal{A}$, and $\mathbb{E}^{0}$ denotes the Palm expectation [17] of the receiver PPP. The fact that the last expression does not depend on the choice of $\mathcal{A}$ results from the stationarity assumptions [17]. Here, $\mathrm{SINR}_{0}$ denotes the SINR measured at the receiver located at the origin 3 . Furthermore, the spectral efficiency of the typical link, or equivalently the spectral efficiency per link is defined by

$$
C_{\text {link }}=\frac{1}{\lambda} C=\mathbb{E}^{0}\left[\sum_{m=1}^{N_{\mathrm{t}, 0}} \log _{2}\left(1+\operatorname{SINR}_{0}(m)\right)\right] .
$$

Here, for the above quantities, we will use the terms ergodic spectral efficiency or ergodic spectral efficiency per link, respectively. The ergodicity is over both the time-domain (averaging over the small-scale multipath fading) and over space (averaging over all Poisson configurations).

We will denote the sum spectral efficiency per unit area by $C^{\mathrm{ZF}}$ under $\mathrm{ZF}$, and by $C^{\mathrm{SIC}}$ undre ZF-SIC, the sum spectral efficiency of $\mathcal{A}$ with ZF by $C_{\mathcal{A}}^{\mathrm{ZF}}$ under ZF and by $C_{\mathcal{A}}^{\mathrm{SIC}}$ under ZF-SIC, and the spectral efficiency per link by $C_{\text {link }}^{\mathrm{ZF}}$ under ZF by $C_{\text {link }}^{\mathrm{SIC}}$ under ZF-SIC.

${ }^{3}$ By Slivnyak's theorem [17], it is possible locate the typical receiver at the origin. We label the typical transmitter and the typical receiver by $X_{0}$ and $Y_{0}=0$, respectively. The distance between $Y_{0}$ and $\left\{X_{k}\right\}_{k \in\{0\} \cup \mathbb{N}}$, the channel matrix $\mathbf{H}_{0, l}, l \in\{0\} \cup \mathbb{N}$, the linear receiver filter $\mathbf{v}_{0}(m), I_{0}(m)$, and the $j$-th nearest interferers from $Y_{0}$, i.e., $X_{0_{j}}$ are defined similarly. 
1) $Z F$ detection: The main idea of the ZF-decorrelator [34] is to construct $\mathbf{v}_{k}(m)$ so as to remove both $I_{k 1}(m)$ and $I_{k 2}(m)$ simultaneously by projecting the received signal vector onto the subspace orthogonal to that spanned by the vectors $\mathbf{H}_{k, k}(:, 1), \ldots, \mathbf{H}_{k, k}(:, m-1), \mathbf{H}_{k, k}($ : $, m+1), \ldots, \mathbf{H}_{k, k}\left(:, N_{\mathrm{t}, k}\right)$, and the column vectors of $\mathbf{H}_{k, k_{1}}, \ldots, \mathbf{H}_{k, k_{L_{k}}}$. Let $\mathbf{U}_{k}(m)$ be the null space of these column vectors; the dimension of $\mathbf{U}_{k}(m)$ is $N_{\mathrm{r}} \times\left(N_{\mathrm{r}}-\left(N_{\mathrm{t}, k}-1\right)-\sum_{i=1}^{L_{k}} N_{\mathrm{t}, k_{i}}\right)$ with probability 14 By definition of $L_{k}, N_{\mathrm{r}}-\left(N_{\mathrm{t}, k}-1\right)-\sum_{i=1}^{L_{k}} N_{\mathrm{t}, k_{i}} \geq 1$.

We are interested in maximizing the desired signal power by choosing $\mathbf{v}_{k}(m)$ in $\mathbf{U}_{k}(m)$. More precisely, we design $\mathbf{v}_{k}(m)$ which maximizes $\left|\mathbf{v}_{k}^{*}(m) \mathbf{H}_{k, k}(:, m)\right|^{2}$. If the columns of $\mathbf{U}_{k}(m)$ are orthonormal bases of the null space, then the following filter maximizes $\left|\mathbf{v}_{k}^{*}(m) \mathbf{H}_{k, k}(:, m)\right|^{2}$ :

$$
\mathbf{v}_{k}(m)=\frac{\mathbf{U}_{k}(m) \mathbf{U}_{k}^{*}(m) \mathbf{H}_{k, k}(:, m)}{\left\|\mathbf{U}_{k}(m) \mathbf{U}_{k}^{*}(m) \mathbf{H}_{k, k}(:, m)\right\|_{2}} .
$$

By applying this filter, $I_{k 1}(m)$ and $I_{k 2}(m)$ are suppressed and the resulting SINR becomes

$$
\operatorname{SINR}_{k}^{Z F}(m)=\frac{H_{k, k}(m) d_{k, k}^{-\alpha}}{I_{k}(m)+\frac{N_{\mathrm{t}, k} \sigma^{2}}{P}}
$$

where $I_{k}(m)=I_{k 3}(m)=\sum_{l, X_{1} \in\left\{\Phi \backslash X_{k}, X_{k_{1}}, X_{k_{2}}, \ldots, X_{k_{L_{k}}}\right\}} H_{k, l} d_{k, l}^{-\alpha}$ and $H_{k, k}(m)=\left\|\mathbf{v}_{k}^{*}(m) \mathbf{H}_{k, k}\right\|^{2}$ is a Chi-squared random variables with $2\left(N_{\mathrm{r}}-N_{\mathrm{t}, k}-\sum_{l=1}^{L_{k}} N_{\mathrm{t}, k_{l}}+1\right)$ degrees of freedom [34] and $H_{k, l}=\left\|\mathbf{v}_{k}^{*}(m) \mathbf{H}_{k, l}\right\|^{2}$ is distributed as a Chi-squared with $2 N_{\mathrm{t}, l}$ degrees of freedom [34]. The sum spectral efficiency per unit area hence becomes

$$
C^{\mathrm{ZF}}=\lambda C_{\text {link }}^{\mathrm{ZF}}=\lambda \mathbb{E}^{0}\left[\sum_{m=1}^{N_{\mathrm{t}, 0}} \log _{2}\left(1+\operatorname{SINR}_{0}^{\mathrm{ZF}}(m)\right)\right] .
$$

2) ZF-SIC detection: We now consider ZF-SIC, which is a well-known non-linear detection method for open-loop MIMO systems. The key idea of ZF-SIC decoding is to recover the data streams successively and to subtract the recovered streams for obtaining the remaining data streams. This provides a power gain as well as an interference cancellation gain. For decoding the data streams of the $k$-th link, the receiver first decodes the signals from interferers using LCSIR. After subtracting off these signals, the $m$-th data of the $k$-th link can be obtained iteratively by decoding and subtracting from the 1 st to the $m-1$-th data streams and by then applying

\footnotetext{
${ }^{4} N_{\mathrm{t}, k}-1$ comes from the dimension of the subspace spanned by $\mathbf{H}_{k, k}(:, 1), \ldots, \mathbf{H}_{k, k}(:, m-1), \mathbf{H}_{k, k}(:, m+1), \ldots, \mathbf{H}_{k, k}($ : ,$\left.N_{\mathrm{t}, k}\right)$ and $\sum_{i=1}^{L_{k}} N_{\mathrm{t}, k_{i}}$ from the dimension of $\mathbf{H}_{k, k_{1}}, \ldots, \mathbf{H}_{k, k_{L_{k}}}$.

${ }^{5}$ The probability density function of the Chi-square distribution with $2 n$ degrees of freedom, $\mathcal{X}_{2 n}^{2}$, is $f_{\mathcal{X}_{2 n}^{2}}(x)=\frac{x^{n-1} e^{-x}}{(n-1) !}$.
} 
the $m$-th decorrelator which suppresses the signal from the $m+1$-th to the $N_{\mathrm{t}, k}$-th streams of the $k$-th link. In other words, the corresponding projection is onto the subspace orthogonal to $\mathbf{H}_{k, k}(:, m+1), \ldots, \mathbf{H}_{k, k}\left(:, N_{\mathrm{t}, k}\right)$ (say $\tilde{\mathbf{U}}_{k}(m)$ ), as opposed to being to the subspace orthogonal to $\mathbf{H}_{k, k}(:, 1), \ldots, \mathbf{H}_{k, k}(:, m-1), \mathbf{H}_{k, k}(:, m+1), \ldots, \mathbf{H}_{k, k}\left(:, N_{\mathrm{t}, k}\right)$ and the column spaces of $\mathbf{H}_{k, k_{1}}, \ldots, \mathbf{H}_{k, k_{L_{k}}}$ in the previous subsection. By choosing $\tilde{\mathbf{v}}_{k}(m)$ in $\tilde{\mathbf{U}}_{k}(m)$ to maximize the signal power, the resulting SINR becomes

$$
\operatorname{SINR}_{k}^{\mathrm{SIC}}(m)=\frac{\tilde{H}_{k, k}(m) d_{k, k}^{-\alpha}}{\tilde{I}_{k}(m)+\frac{N_{\mathrm{t}, k} \sigma^{2}}{P}},
$$

where $\tilde{I}_{k}(m)=I_{k 3}(m)=\sum_{l, X_{l} \in\left\{\Phi \backslash X_{k}, X_{k_{1}}, X_{k_{2}}, \ldots, X_{k_{L_{k}}}\right\}} \tilde{H}_{k, l} d_{k, l}^{-\alpha}, \tilde{H}_{k, k}(m)=\left\|\tilde{\mathbf{v}}_{k}^{*}(m) \mathbf{H}_{k, k}\right\|^{2}$ is a Chi-squared random variable with $2\left(N_{\mathrm{r}}-N_{\mathrm{t}, k}+m\right)$ degrees of freedom and $\tilde{H}_{k, l}=\left\|\tilde{\mathbf{v}}_{k}^{*}(m) \mathbf{H}_{k, l}\right\|^{2}$ is distributed as a Chi-squared with $2 N_{\mathrm{t}, l}$ degrees of freedom. 6 The sum spectral efficiency per unit area achieved by the ZF-SIC is given by

$$
C^{\mathrm{SIC}}=\lambda C_{\text {link }}^{\mathrm{SIC}}=\lambda \mathbb{E}^{0}\left[\sum_{m=1}^{N_{\mathrm{to}}} \log _{2}\left(1+\operatorname{SINR}_{0}^{\mathrm{SIC}}(m)\right)\right] .
$$

Even though neither ZF nor ZF-SIC are optimal in the information theoretic sense, these are quite commonly used and in addition amenable to analysis. With these receiving architectures, the exact expressions of the sum spectral efficiency and the corresponding scaling laws are given in the following sections.

\section{DIRECT CSIR}

In this section, we obtain the exact analytical expressions of the sum spectral efficiency for both ZF and ZF-SIC detection with DCSIR, i.e., $L_{k}=0$ for all $X_{k} \in \Phi$. Then, we derive a lower and an upper bounds with closed-forms. We get the announced scaling laws from these closed from expressions.

In our closed-form expressions, we use the Gamma function which is defined as $\Gamma(x)=$ $\int_{0}^{\infty} t^{x-1} e^{-t} d t$

${ }^{6}$ With the SIC structure, the subspace spanned by $\mathbf{H}_{k, k}(:, m+1), \ldots, \mathbf{H}_{k, k}\left(:, N_{\mathrm{t}, k}\right)$ is suppressed for recovering the $m$-th data stream. 


\section{A. Sum Spectral Efficiency}

Theorem 1 (ZF with DCSIR): When using ZF detection, the sum spectral efficiency per unit area of DCSIR is

$$
\begin{aligned}
C^{\mathrm{ZF}=} & \left.\left.\sum_{v=1}^{N_{\mathrm{r}}} \frac{\alpha \lambda v p_{v}}{2 \ln 2} \int_{1}^{R_{d}} \int_{0}^{\infty} \frac{e^{-\frac{v \sigma_{r}^{2} \alpha}{P}\left(\lambda \pi \sum_{k=1}^{N_{\mathrm{r}}} p_{k} \frac{\Gamma\left(k+\frac{2}{\alpha}\right) \Gamma\left(1-\frac{2}{\alpha}\right)}{\Gamma(k) u}\right)^{-\frac{\alpha}{2}}-u}}{u}\right)^{N_{\mathrm{r}}-v+1}\right) d u \frac{2 r}{R_{d}^{2}-1} d r . \\
& \left(1-\left(\frac{1}{1+\left(\lambda \pi r^{2} \sum_{k=1}^{N_{\mathrm{r}}} p_{k} \frac{\Gamma\left(k+\frac{2}{\alpha}\right) \Gamma\left(1-\frac{2}{\alpha}\right)}{\Gamma(k) u}\right)^{-\frac{\alpha}{2}}}\right)^{(1)}\right.
\end{aligned}
$$

Proof: See Appendix B

Theorem 2 (ZF-SIC with DCSIR): When using ZF-SIC detection, the sum spectral efficiency per unit area of DCSIR is

$$
\begin{aligned}
C^{\mathrm{SIC}}= & \left.\left.\sum_{v=1}^{N_{\mathrm{r}}}\left[\frac{\alpha \lambda p_{v}}{2 \ln 2} \int_{1}^{R_{d}} \int_{0}^{\infty} \frac{e^{-\frac{v \sigma^{2} r^{\alpha}}{P}}\left(\lambda \pi \sum_{k=1}^{N_{\mathrm{r}}} p_{k} \frac{\Gamma\left(k+\frac{2}{\alpha}\right) \Gamma\left(1-\frac{2}{\alpha}\right)}{\Gamma(k) u}\right)^{-\frac{\alpha}{2}}-u}{u}\right)^{N_{\mathrm{r}}-v+m}\right) d u \frac{2 r}{R_{d}^{2}-1} d r\right] . \\
& \sum_{m=1}^{v}\left(1-\left(\frac{1}{1+\left(\lambda \pi r^{2} \sum_{k=1}^{N_{\mathrm{r}}} p_{k} \frac{\Gamma\left(k+\frac{2}{\alpha}\right) \Gamma\left(1-\frac{2}{\alpha}\right)}{\Gamma(k) u}\right)^{-\frac{\alpha}{2}}}\right)^{.}\right.
\end{aligned}
$$

Proof: See Appendix B

Corollary 1: When all transmitters have $N_{\mathrm{t}}$ antennas, i.e. $p_{N_{\mathrm{t}}}=1$, (13) simplifies to

$$
\begin{aligned}
C^{\mathrm{ZF}=}= & \left.\left.\frac{\alpha \lambda N_{\mathrm{t}}}{2 \ln 2} \int_{1}^{R_{d}} \int_{0}^{\infty} \frac{e^{-\frac{N_{\mathrm{t}} \sigma^{2} r^{\alpha}}{P}\left(\lambda \pi \frac{\Gamma\left(N_{\mathrm{t}}+\frac{2}{\alpha}\right) \Gamma\left(1-\frac{2}{\alpha}\right)}{\Gamma\left(N_{\mathrm{t}}\right) u}\right)^{-\frac{\alpha}{2}}-u}}{u}\right)^{N_{\mathrm{r}}-N_{\mathrm{t}}+1}\right) d u \frac{2 r}{R_{d}^{2}-1} d r, \\
& \left(1-\left(\frac{1}{1+\left(\lambda \pi r^{2} \frac{\Gamma\left(N_{\mathrm{t}}+\frac{2}{\alpha}\right) \Gamma\left(1-\frac{2}{\alpha}\right)}{\Gamma\left(N_{\mathrm{t}}\right) u}\right)^{-\frac{\alpha}{2}}}\right)^{(1)}\right.
\end{aligned}
$$

and (14) reduces to

$$
\begin{aligned}
C^{\mathrm{SIC}}= & \frac{\alpha \lambda}{2 \ln 2} \int_{1}^{R_{d}} \int_{0}^{\infty} \frac{e^{-\frac{N_{\mathrm{t}} \sigma^{2} r^{\alpha}}{P}\left(\lambda \pi \frac{\Gamma\left(N_{\mathrm{t}}+\frac{2}{\alpha}\right) \Gamma\left(1-\frac{2}{\alpha}\right)}{\Gamma\left(N_{\mathrm{t}}\right) u}\right)^{-\frac{\alpha}{2}}-u}}{u} \\
& \sum_{m=1}^{N_{\mathrm{t}}}\left(1-\left(\frac{1}{1+\left(\lambda \pi r^{2} \frac{\Gamma\left(N_{\mathrm{t}}+\frac{2}{\alpha}\right) \Gamma\left(1-\frac{2}{\alpha}\right)}{\Gamma\left(N_{\mathrm{t}}\right) u}\right)^{-\frac{\alpha}{2}}}\right) d u \frac{2 r}{R_{d}^{2}-1} d r .\right.
\end{aligned}
$$




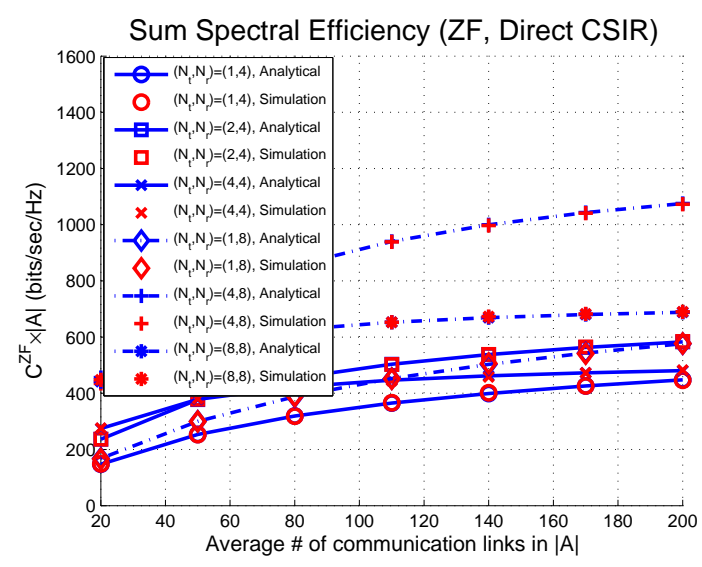

(a) ZF detection

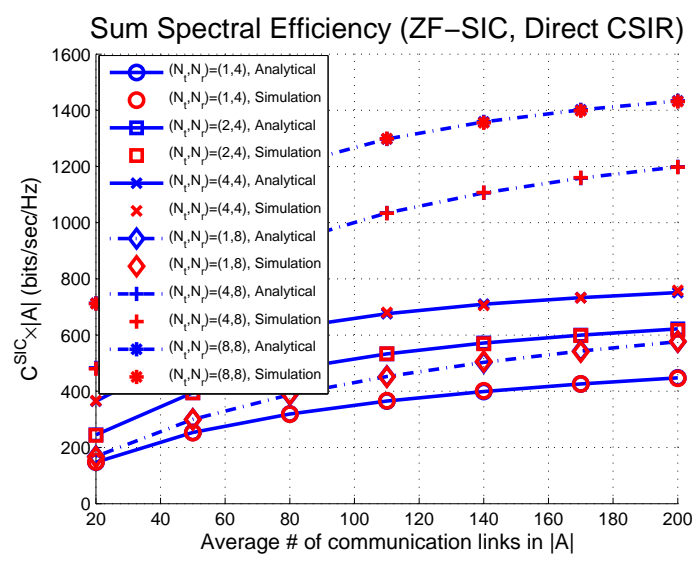

(b) ZF-SIC detection

Fig. 2: The sum efficiency with DCSIR when $|\mathcal{A}|=\pi 500^{2}\left(m^{2}\right), \alpha=4, R_{d}=50(m), P=-20(d B m)$, $\sigma^{2}=-104(d B m), p_{N_{\mathrm{t}}}=1$

These explicit formulas show how the sum spectral efficiency is determined by the system parameters. Fig. 2 plots the sum spectral efficiency of transmitters in region $\mathcal{A} \subset \mathbb{R}^{2}$ for a path-loss exponent $\alpha=4,|\mathcal{A}|=\pi 500^{2}\left(m^{2}\right), R_{d}=50 \mathrm{~m}, P=-20 \mathrm{dBm}, p_{N_{\mathrm{t}}}=1$ and $\sigma^{2}=$ $-104 \mathrm{dBm}$. The gain of the SIC decorrelator can be found by comparing the two figures in Fig. 2 ,

Remark 1: A drawback of ZF-SIC is error propagation. In the high SNR regime, however, ZF-SIC detection, which is based on a higher-dimensional subspace, always provides a higher sum spectral efficiency than ZF detection, as can be checked in Fig. 2.

One of the interesting observations is that increasing the number of streams $N_{\mathrm{t}}$ for a given $N_{\mathrm{r}}$ and $\lambda$ does not guarantee increasing the sum spectral efficiency. On the one hand, for a small node density $\lambda$, it is possible to increase the sum spectral efficiency linearly with the number of spatial multiplexing streams $N_{\mathrm{t}}$. On the other hand, when $\lambda$ is large enough, it is not optimal to send $N_{\mathrm{t}}$ data streams, as the sum spectral efficiency increases sub-linearly with $\lambda$ as shown in Fig. 2. This implies that, for fixed $N_{\mathrm{t}}$ and $N_{\mathrm{r}}$, there exists an optimal density of nodes which maximizes the sum spectral efficiency per link in such a network. To further obtain insights from the derived expressions, it is instructive to consider some examples:

Example 1: When $d_{k, k}=d$ for all $k \in \mathcal{K}$ and $p_{N_{\mathrm{t}}}=1$, Equations (15) and (16) can be 
simplified as follows

$$
\begin{aligned}
C^{\mathrm{ZF}} & =\frac{\lambda \alpha N_{\mathrm{t}}}{2 \ln 2} \sum_{n=1}^{N_{\mathrm{r}}-N_{\mathrm{t}}+1}\left(\begin{array}{c}
N_{\mathrm{r}}-N_{\mathrm{t}}+1 \\
n
\end{array}\right) \int_{0}^{\infty} \frac{e^{-u}}{u} \frac{\left(\frac{\Gamma\left(N_{\mathrm{t}}\right) u}{\lambda \Gamma\left(N_{\mathrm{t}}+\frac{2}{\alpha}\right) \Gamma\left(1-\frac{2}{\alpha}\right) \pi d^{2}}\right)^{n \frac{\alpha}{2}}}{\left(1+\left(\frac{\Gamma\left(N_{\mathrm{t}}\right) u}{\lambda \Gamma\left(N_{\mathrm{t}}+\frac{2}{\alpha}\right) \Gamma\left(1-\frac{2}{\alpha}\right) \pi d^{2}}\right)^{\frac{\alpha}{2}}\right)^{N_{\mathrm{r}}-N_{\mathrm{t}}+1}} d u \\
C^{\mathrm{SIC}} & =\frac{\lambda \alpha}{2 \ln 2} \sum_{m=1}^{N_{\mathrm{t}}} \sum_{n=1}^{N_{\mathrm{r}}-N_{\mathrm{t}}+m}\left(\begin{array}{c}
N_{\mathrm{r}}-N_{\mathrm{t}}+m \\
n
\end{array}\right) \int_{0}^{\infty} \frac{e^{-u}}{u} \frac{\left(\frac{\Gamma\left(N_{\mathrm{t}}\right) u}{\lambda \Gamma\left(N_{\mathrm{t}}+\frac{2}{\alpha}\right) \Gamma\left(1-\frac{2}{\alpha}\right) \pi d^{2}}\right)^{n \frac{\alpha}{2}}}{\left(1+\left(\frac{\Gamma\left(N_{\mathrm{t}}\right) u}{\lambda \Gamma\left(N_{\mathrm{t}}+\frac{2}{\alpha}\right) \Gamma\left(1-\frac{2}{\alpha}\right) \pi d^{2}}\right)^{\frac{\alpha}{2}}\right)^{N_{\mathrm{r}}-N_{\mathrm{t}}+m}} d u,
\end{aligned}
$$

in the interference-limited case $\left(\sigma^{2}=0\right)$. This simplified single integral form provides a better intuition on the impact of network design parameters on sum spectral efficiency. For example, increasing $N_{\mathrm{r}}$ always provides higher performance, and optimizing $N_{\mathrm{t}}$ for fixed $N_{\mathrm{r}}$ is an important and interesting question.

Example 2: Following the Example 1, we further assume that $N_{\mathrm{t}}=N_{\mathrm{r}}, \alpha=4$. In this case, the sum spectral efficiency per unit area with the ZF-receiver is

$$
\begin{aligned}
C^{\mathrm{ZF}} & =\frac{2 \lambda N_{\mathrm{t}}}{\ln 2}\left\{\sin \left(\frac{\pi \lambda d^{2} \Gamma\left(N_{\mathrm{t}}+\frac{1}{2}\right) \Gamma\left(\frac{1}{2}\right)}{\Gamma\left(N_{\mathrm{t}}\right)}\right)\left(\frac{\pi}{2}-S i\left(\frac{\pi \lambda d^{2} \Gamma\left(N_{\mathrm{t}}+\frac{1}{2}\right) \Gamma\left(\frac{1}{2}\right)}{\Gamma\left(N_{\mathrm{t}}\right)}\right)\right)\right. \\
& \left.-\cos \left(\frac{\pi \lambda d^{2} \Gamma\left(N_{\mathrm{t}}+\frac{1}{2}\right) \Gamma\left(\frac{1}{2}\right)}{\Gamma\left(N_{\mathrm{t}}\right)}\right) C i\left(\frac{\pi \lambda d^{2} \Gamma\left(N_{\mathrm{t}}+\frac{1}{2}\right) \Gamma\left(\frac{1}{2}\right)}{\Gamma\left(N_{\mathrm{t}}\right)}\right)\right\},
\end{aligned}
$$

where $\operatorname{Si}(z)=\int_{0}^{z} \frac{\sin (t)}{t} d t$ and $C i(z)=-\int_{z}^{\infty} \frac{\cos (t)}{t} d t$ are the sine integral and cosine integral functions.

In Example 2, if we assume $d=\sqrt{\frac{\Gamma\left(N_{\mathrm{t}}\right)}{2 \lambda \Gamma\left(N_{\mathrm{t}}+\frac{1}{2}\right) \Gamma\left(\frac{1}{2}\right)}}$, which means that the distance of communication links is of order of $\lambda^{-\frac{1}{2}}$, the sum spectral efficiency per unit area becomes

$$
C^{\mathrm{ZF}}=\frac{2 \lambda N_{\mathrm{t}}}{\ln 2}\left(\frac{\pi}{2}-S i\left(\frac{\pi}{2}\right)\right) \simeq 0.5772 \lambda N_{\mathrm{t}} .
$$

So, if the assumptions in Example 2 and the above relation of $d$ and $\lambda$ hold, it is possible to guarantee that the sum spectral efficiency per unit area is at least $0.5772 N_{\mathrm{t}} \lambda$ by choosing $N_{\mathrm{t}}$ equal to $N_{\mathrm{r}}$.

Throughout this paper, the main scaling is that of the number of transmit and receive antennas with respect to the network density $\lambda$. This example different from the main stream as the link distance depends on the network density $\lambda$. In what follows link distances will not exhibit such a functional depencency. 


\section{B. Scaling Law}

In this section, we provide both a lower and an upper bound with a closed-form on the sum spectral efficiency. This allows us to obtain the announced scaling law. We focus on the case where $p_{N_{\mathrm{t}}}=1$.

Theorem 3 (Direct CSIR, ZF, Scaling Law): Assume that $p_{N_{\mathrm{t}}}=1, N_{\mathrm{t}}=c_{1} \lambda^{\beta_{1}}, N_{\mathrm{r}}=c_{2} \lambda^{\beta_{2}}$, for some constants $c_{1}, c_{2}>0$, and that $\beta_{1} \leq \beta_{2}$. Then, in the interference limited regime,

$$
\lim _{\lambda \rightarrow \infty} C^{\mathrm{ZF}}=\Theta\left(\lambda^{\beta_{1}+1} \log _{2}\left(1+\lambda^{\beta_{2}-\beta_{1}-\frac{\alpha}{2}}\right)\right)
$$

Proof: See Appendix C.

Theorem 4 (Direct CSIR, Scaling Law, ZF-SIC): Under the same assumptions as in Theorem 3, in the interference limited regime,

$$
\lim _{\lambda \rightarrow \infty} C^{\mathrm{SIC}}=\Theta\left(\lambda^{\beta_{1}+1} \log _{2}\left(1+\lambda^{\beta_{2}-\beta_{1}-\frac{\alpha}{2}}\right)\right) .
$$

Proof: See Appendix [C]

Remark 2: The first observation is that, in the DCSIR case, the sum spectral efficiency per unit area are identical for ZF and ZF-SIC in a scaling law sense. This is because the signal power gain under ZF-SIC is at most $N_{\mathrm{t}}$, i.e., $\mathbb{E}\left[H_{k, k}(m)\right] \simeq N_{\mathrm{t}}$, while the fading power of inter-

node interference is also proportional to $\frac{1}{N_{\mathrm{t}}}$, i.e., $\left.\left(\mathbb{E}\left[\frac{1}{I_{k}(m)}\right]\right] \simeq \frac{1}{N_{\mathrm{t}}}\right)$. Consequently, the array gain obtained by ZF-SIC detection is negligible in the scaling law sense. To obtain a gain from the SIC structure, the signal power gain by ZF-SIC should be larger than $N_{\mathrm{t}}$, and this will actually be the case for LCISR (see Section IV]).

The next corollary, on per link spectral efficiency, follows immediately from the two theorems stated above.

Corollary 2: When the receive scheme is ZF or ZF-SIC, under DCSIR, the scaling law of the sum spectral efficiency per link is

$$
\begin{array}{ll}
\Theta\left(\lambda^{\beta_{1}} \log (\lambda)\right) & \text { for } \beta_{2}-\beta_{1}-\frac{\alpha}{2}>0, \\
\Theta\left(\lambda^{\beta_{1}}\right) & \text { for } \beta_{2}-\beta_{1}-\frac{\alpha}{2}=0, \\
\Theta\left(\lambda^{\beta_{2}-\frac{\alpha}{2}}\right) & \text { for } \beta_{2}-\beta_{1}-\frac{\alpha}{2}<0 .
\end{array}
$$

Here are important observations following from this corollary. 
- Whenever $\beta_{2}-\beta_{1}-\frac{\alpha}{2} \geq 0$, the spectral efficiency per link is determined by $N_{\mathrm{t}}$ alone. So, in this regime, spatial multiplexing, namely increasing the number of data streams, is beneficial; to the best of our knowledge, this result is new.

- Whenever $\beta_{2}-\beta_{1}-\frac{\alpha}{2}<0$, the sum spectral efficiency per unit area goes to 0 exponentially fast with $\lambda$ when $\beta_{2}<\frac{\alpha}{2}$, and increases like $\lambda^{\beta_{2}-\frac{\alpha}{2}}$ when $\beta_{2}>\frac{\alpha}{2}$. For given $\beta_{2}$ and $\alpha$ with $\beta_{2}-\frac{\alpha}{2}>0$, the best value for $\beta_{1}$ is $\beta_{1}^{*}=\beta_{2}-\frac{\alpha}{2}$, and the corresponding scaling law is $\Theta\left(\lambda^{\beta_{2}-\frac{\alpha}{2}}\right)$.

- We can expect a linear gain when $\beta_{2}=\frac{\alpha}{2}$ as this is the critical region between the superlinear and sub-linear regions.

- For fixed $N_{\mathrm{t}}$ and $N_{\mathrm{r}}$, (i.e. $\beta_{1}, \beta_{2}=0$ ), the scaling law is $\Theta\left(\lambda^{-\frac{\alpha}{2}}\right)$.

Example 3: Assume that $p_{N_{\mathrm{t}}}=1$. For fixed values of $N_{\mathrm{t}}, N_{\mathrm{r}}$ and $\alpha$, what is the optimal node density in our model? We answer this question in a heuristic way by maximizing the lower bounds obtained above. For the ZF case, the density maximizing the lower bound of the sum spectral efficiency per unit area in (62) is 7

$$
\lambda_{\mathrm{ZF}}^{*}=\arg \max _{\lambda} \frac{2 \lambda N_{\mathrm{t}}}{\alpha} \log _{2}\left(1+\left(\frac{2 \Gamma\left(N_{\mathrm{t}}\right)}{\Gamma\left(N_{\mathrm{t}}+\frac{2}{\alpha}\right) \Gamma\left(1-\frac{2}{\alpha}\right)}\right)^{\frac{\alpha}{2}} \frac{N_{\mathrm{r}}-N_{\mathrm{t}}}{\left(\lambda \pi\left(R_{d}^{2}+1\right)\right)^{\frac{\alpha}{2}}}\right) .
$$

For large $x, \log _{2}(1+x) \simeq \log _{2}(x)$, so in the high SIR regime, the optimal link density is

$$
\lambda_{\mathrm{ZF}}^{*}=\frac{\Gamma\left(N_{\mathrm{t}}\right)}{2^{\ln 2-1} \Gamma\left(N_{\mathrm{t}}+\frac{2}{\alpha}\right) \Gamma\left(1-\frac{2}{\alpha}\right)} \frac{\left(N_{\mathrm{r}}-N_{\mathrm{t}}\right)^{\frac{2}{\alpha}}}{\pi\left(R_{d}^{2}+1\right)} .
$$

Hence, the optimal probability in the Aloha protocol for a given $\lambda, N_{\mathrm{t}}, N_{\mathrm{r}}, \alpha$ is

$$
p_{\mathrm{ZF}}^{*}=\min \left(1, \frac{\lambda_{\mathrm{ZF}}^{*}}{\lambda}\right) \text {. }
$$

For the ZF-SIC case, by using the lower bound in (71) and the relation $\log _{2}(1+x) \simeq \log _{2}(x)$ for large $x$, we get that the optimal $\lambda$ given $N_{\mathrm{t}}, N_{\mathrm{r}}$, and $\lambda$ in high SIR regime is

$$
\begin{aligned}
\lambda_{\mathrm{SIC}}^{*} & =\arg \max _{\lambda} \frac{2 \lambda}{\alpha} \sum_{m=1}^{N_{\mathrm{t}}} \log _{2}\left(1+\left(\frac{2 \Gamma\left(N_{\mathrm{t}}\right)}{\Gamma\left(N_{\mathrm{t}}+\frac{2}{\alpha}\right) \Gamma\left(1-\frac{2}{\alpha}\right)}\right)^{\frac{\alpha}{2}} \frac{N_{\mathrm{r}}-N_{\mathrm{t}}+m-1}{\left(\lambda \pi\left(R_{d}^{2}+1\right)\right)^{\frac{\alpha}{2}}}\right) \\
& \simeq \arg \max _{\lambda} \frac{2 \lambda}{\alpha} \log _{2}\left(\left(\frac{2 \Gamma\left(N_{\mathrm{t}}\right)}{\Gamma\left(N_{\mathrm{t}}+\frac{2}{\alpha}\right) \Gamma\left(1-\frac{2}{\alpha}\right) \pi\left(R_{d}^{2}+1\right)}\right)^{\frac{\alpha N_{\mathrm{t}}}{2}} \prod_{m=1}^{N_{\mathrm{t}}}\left(N_{\mathrm{r}}-N_{\mathrm{t}}+m-1\right) \lambda^{-\frac{\alpha N_{\mathrm{t}}}{2}}\right) \\
& =\frac{\Gamma\left(N_{\mathrm{t}}\right)}{2^{\ln 2-1} \Gamma\left(N_{\mathrm{t}}+\frac{2}{\alpha}\right) \Gamma\left(1-\frac{2}{\alpha}\right) \pi\left(R_{d}^{2}+1\right)}\left(\prod_{m=1}^{N_{\mathrm{t}}}\left(N_{\mathrm{r}}-N_{\mathrm{t}}+m-1\right)\right)^{\frac{2}{N_{\mathrm{t}} \alpha}}
\end{aligned}
$$

${ }^{7}$ Here, we ignore $\epsilon$. 
and the optimal Aloha probability is

$$
p_{\mathrm{SIC}}^{*}=\min \left(1, \frac{\lambda_{\mathrm{SIC}}^{*}}{\lambda}\right) .
$$

Example 4: Assume $p_{N_{\mathrm{t}}}=1$. For fixed $N_{\mathrm{r}}, \lambda$, and $\alpha$, What is the optimal value for $N_{\mathrm{t}}$ ? This can be obtained by using the formulas in Theorem 1 and 2. A simple way consists in maximizing the lower bounds as in Example 3. By using the Gamma function relation

$$
\left(\frac{\Gamma\left(N_{\mathrm{t}}\right)}{\Gamma\left(N_{\mathrm{t}}+\frac{2}{\alpha}\right) \Gamma\left(1-\frac{2}{\alpha}\right)}\right)^{\frac{\alpha}{2}} \geq \frac{1}{N_{\mathrm{t}}},
$$

Equation (62), which is the lower bound of sum spectral efficiency per unit area when ZF-receiver is applied, becomes

$$
\frac{2 \lambda N_{\mathrm{t}}}{\alpha} \log _{2}\left(1+b \frac{N_{\mathrm{r}}-N_{\mathrm{t}}}{N_{\mathrm{t}}}\right)
$$

when we define

$$
b \triangleq\left(\frac{2}{\Gamma\left(1-\frac{2}{\alpha}\right)}\right)^{\frac{\alpha}{2}} \frac{1}{\left(\lambda \pi R_{d}^{2}\right)^{\frac{\alpha}{2}}} .
$$

In the high SIR regime, the optimal $N_{\mathrm{t}}$ for maximizing (32) is

$$
N_{\mathrm{t}, \mathrm{ZF}}^{*}=\frac{b N_{\mathrm{r}}}{e} .
$$

In the same manner, we can obtain that the value of $N_{\mathrm{t}}$ maximizing (71) when ZF-SIC is applied is

$$
N_{\mathrm{t}, \mathrm{SIC}}^{*}=N_{\mathrm{t}, \mathrm{ZF}}^{*}=\frac{b N_{\mathrm{r}}}{e} .
$$

\section{LOCAL CSIR}

As already explained, LCSIR denotes the situation where $L_{k}>0$, i.e. receiver $k$ knows the $L_{k}$-nearest interferer CSIs in addition to the CSI of its own channel. Through this section, we assume all transmitters are equipped with $N_{\mathrm{t}}$ antennas (i.e., $p_{N_{\mathrm{t}}}=1$ ) and $L_{k}=L$ for all $X_{k} \in \Phi$, consequently $1 \leq L \leq\left\lfloor\frac{N_{\mathrm{r}}}{N_{\mathrm{t}}}\right\rfloor-1$. 


\section{A. Sum Spectral Efficiency}

In the LCSIR case, we denote the sum spectral efficiency per unit area by $C_{L}^{\mathrm{ZF}}$ under ZF and by $C_{L}^{\mathrm{SIC}}$ under ZF-SIC.

Theorem 5: In the LCSIR case, under ZF detection, the achievable sum spectral efficiency per unit area with $L$ dominant interferer CSI is

$$
C_{L}^{\mathrm{ZF}}=\frac{\lambda N_{\mathrm{t}}}{\ln 2} \int_{1}^{R_{d}} \int_{0}^{\infty} \frac{1}{s e^{\frac{N_{\mathrm{t}} \sigma^{2} s}{P}}}\left(1-\frac{1}{\left(1+s x^{-\alpha}\right)^{N_{\mathrm{r}}-(L+1) N_{\mathrm{t}}+1}}\right) \mathcal{L}_{\tilde{I}_{k}}(L ; s) d s \frac{2 x}{R_{d}^{2}} d x,
$$

where

$$
\mathcal{L}_{\tilde{I}_{k}}(L ; s)=\int_{0}^{\infty} \exp \left(-\pi \lambda \int_{u=r^{2}}^{\infty} 1-\left(\frac{1}{1+s u^{-\frac{\alpha}{2}}}\right)^{N_{\mathrm{t}}} d u\right) \frac{2\left(\lambda \pi r^{2}\right)^{L}}{r \Gamma(L)} e^{-\lambda \pi r^{2}} d r .
$$

Proof: See Appendix D,

Theorem 6: In the local CSIR case, the achievable sum spectral efficiency per unit area with $L$ dominant interferer channel information using ZF-SIC detection is

$$
C_{L}^{\mathrm{SIC}}=\sum_{m=1}^{N_{\mathrm{t}}} \frac{\lambda}{\ln 2} \int_{1}^{R_{d}} \int_{0}^{\infty} \frac{1}{s e^{\frac{N_{\mathrm{t}} \sigma_{s} s}{P}}}\left(1-\frac{1}{\left(1+s x^{-\alpha}\right)^{N_{\mathrm{r}}-N_{\mathrm{t}}+m}}\right) \mathcal{L}_{\tilde{I}_{k}}(L ; s) d s \frac{2 x}{R_{d}^{2}} d x,
$$

where

$$
\mathcal{L}_{\tilde{I}_{k}}(L ; s)=\int_{r=0}^{\infty} \exp \left(-\pi \lambda \int_{u=r^{2}}^{\infty} 1-\left(\frac{1}{1+s u^{-\frac{\alpha}{2}}}\right)^{N_{\mathrm{t}}} d u\right) \frac{2\left(\lambda \pi r^{2}\right)^{L}}{r \Gamma(L)} e^{-\lambda \pi r^{2}} d r .
$$

Proof: See Appendix D,

Here as in the DCSIR case, the sum spectral efficiency increases with the network density, for both ZF and ZF-SIC. This can be checked in Fig. 3 where we see that the sum capacity increases sub-linearly with the average number of links.

Remark 3: For the ZF case, the fading power of the desired signal is lower for LCSIR than for DCSIR because the remaining degrees of freedom at the receiver are used to suppress the internode interference from the $L$-dominant interferers. Therefore, leveraging all channel information is not always beneficial. This can be checked in the first figures of Fig. 2 and Fig. 3, For the ZF-SIC case, however, utilizing all information is always beneficial, since the fading power of the $m$-th data stream of the $k$-th link is $\chi_{2\left(N_{\mathrm{r}}-N_{\mathrm{t}}+m\right)}^{2}$, rather than $\chi_{2\left(N_{\mathrm{r}}-(L+1) N_{\mathrm{t}}+1\right)}^{2}$ in ZF. This observation can be checked on the second figures of Fig. 2 and Fig. 3 , 


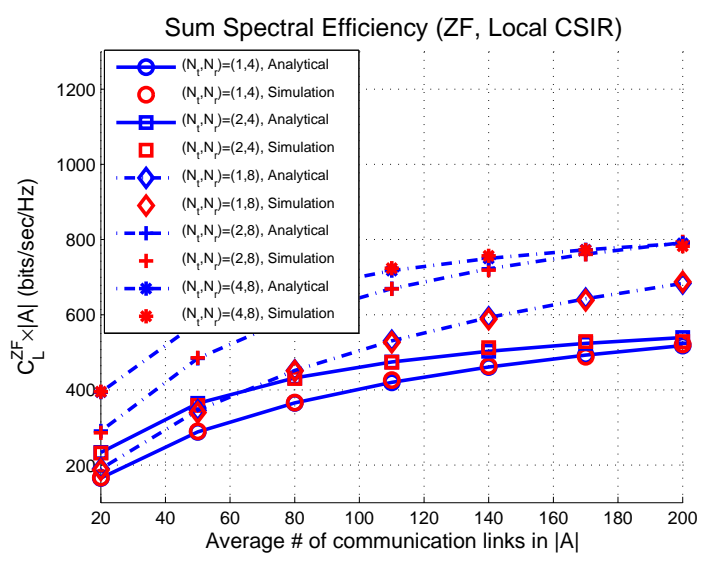

(a) ZF detection

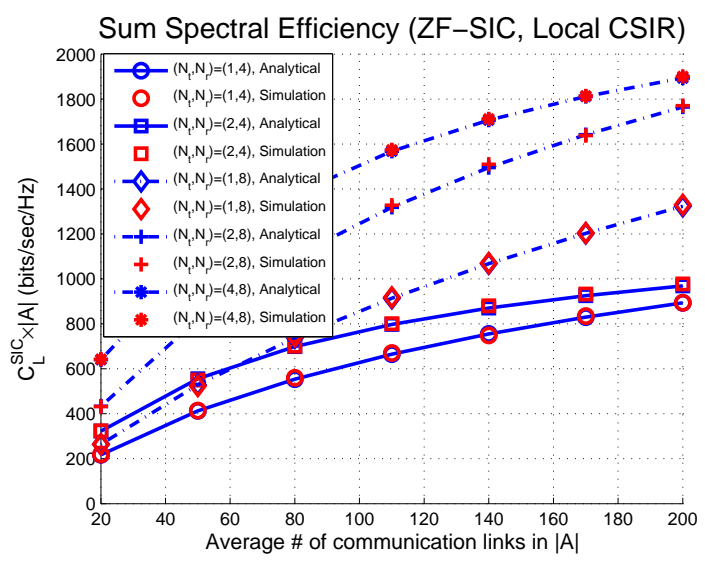

(b) ZF-SIC detection

Fig. 3: The sum spectral efficiency with LCSIR when $|\mathcal{A}|=\pi 500^{2}\left(m^{2}\right), \alpha=4, R_{d}=50(m), P=-20(d B m)$, $\sigma^{2}=-104(d B m)$ with $L=\left\lfloor\frac{N_{r}}{N_{t}}\right\rfloor-1$.

\section{B. Scaling Law}

In this section, we assume that $L=\left\lfloor\frac{N_{\mathrm{r}}}{N_{\mathrm{t}}}\right\rfloor-1$ which is the maximum possible number for nulling the interference from other nodes.

Theorem 7 (Local CSIR, Scaling Law, ZF): Assume that $p_{N_{\mathrm{t}}}=1$, and $N_{\mathrm{t}}=c_{1} \lambda^{\beta_{1}}, N_{\mathrm{r}}=$ $c_{2} \lambda^{\beta_{2}}$ with with some constants $c_{1}, c_{2}>0$ and $\beta_{1} \leq \beta_{2}$. Then, under ZF detection, the sum spectral efficiency per unit area scales as

$$
\lim _{\lambda \rightarrow \infty} C_{L}^{\mathrm{ZF}}=\Omega\left(\lambda^{\beta_{1}+1} \log _{2}\left(1+\lambda^{\left(\beta_{2}-\beta_{1}-1\right) \frac{\alpha}{2}-\beta_{2}}\right)\right)
$$

when $L=\left\lfloor\frac{N_{\mathrm{r}}}{N_{\mathrm{t}}}\right\rfloor-1$.

Proof: See Appendix E,

Remark 4: When $\alpha=4$, under ZF, the scaling law of spectral efficiency per link is $\Theta\left(\lambda^{\beta_{1}} \log _{2}(1+\right.$ $\left.\left.\lambda^{\beta_{2}-\beta_{1}-1}\right)\right)$ for DCSIR, whereas it is $\Omega\left(\lambda^{\beta_{1}} \log _{2}\left(1+\lambda^{\beta_{2}-2 \beta_{1}-2}\right)\right)$ for LCSIR In this case, we can observe that knowing channel state from other nodes is not useful in the sense of scaling laws. This is because the receiver wastes the spatial degrees of freedom to cancel the nearest internode interference. We conclude that, when ZF detection is employed, in the scaling law sense, treating the nearest inter-node interference as noise is a better strategy than canceling it.

Theorem 8 (Local CSIR, Scaling Law, ZF-SIC): The assumptions for the number of antenna configurations are the same as in Theorem 7. When $L=\left\lfloor\frac{N_{\mathrm{r}}}{N_{\mathrm{t}}}\right\rfloor-1$, the sum spectral efficiency 
per unit area with ZF-SIC detection scales as

$$
\lim _{\lambda \rightarrow \infty} C_{L}^{\mathrm{SIC}}=\Omega\left(\lambda^{\beta_{1}+1} \log _{2}\left(1+\lambda^{\left(\beta_{2}-\beta_{1}-1\right) \frac{\alpha}{2}}\right)\right) .
$$

Proof: See Appendix E.

The main difference between (40) and (41) is the degrees of freedom of signal power by the successive cancellation architecture.

Corollary 3: Under ZF-SIC and LCSIR, the scaling law of the ergodic spectral efficiency per link is

$$
\begin{array}{lr}
\Omega\left(\lambda^{\beta_{1}} \log (\lambda)\right) & \text { for } \beta_{2}-\beta_{1}-1>0 \\
\Omega\left(\lambda^{\beta_{1}}\right) & \text { for } \beta_{2}-\beta_{1}-1=0 \\
\Omega\left(\lambda^{\beta_{1}+\left(\beta_{2}-\beta_{1}-1\right) \frac{\alpha}{2}}\right) & \text { for } \beta_{2}-\beta_{1}-1<0 .
\end{array}
$$

The conclusions are similar to those of Corollary 2. In particular, for given $\beta_{2}$ and $\alpha$, the best $\beta_{1}$ in the scaling law sense is hence $\beta_{1}^{*}=\beta_{2}-1$, and the corresponding scaling law is $\Omega\left(\lambda^{\beta_{2}-1}\right)$. Since we assume $\alpha>2$, by comparing with the scaling law in Corollary 2, LCSIR can achieve higher performance than DCSIR case in the ergodic spectral efficiency per link scaling law sense.

Example 5: When $N_{\mathrm{t}}, N_{\mathrm{r}}, \lambda$, and $L$ are given, the density maximizing the lower bounds in (83) for ZF and (85) for ZF-SIC under LCSIR can be obtained as follows. As in Example 3, in the high SIR regime, the optimal densities for ZF and ZF-SIC are

$$
\begin{aligned}
\lambda_{\mathrm{ZF}, \mathrm{L}}^{*} & =\left(\frac{N_{\mathrm{r}}-(L+1) N_{\mathrm{t}}}{\frac{2\left(1-R_{d}^{2-\alpha}\right)}{(\alpha-2)\left(R_{d}^{2}-1\right)}(2 \pi)^{\frac{\alpha}{2}} N_{\mathrm{t}}}\left(L-\frac{\alpha}{2}\right)^{\frac{\alpha}{2}-1}\right)^{\frac{2}{\alpha}} \frac{1}{2^{\ln 2}} \\
\lambda_{\mathrm{SIC}, \mathrm{L}}^{*} & =\left(\frac{\left(L-\frac{\alpha}{2}\right)^{\frac{\alpha}{2}-1}}{\frac{2\left(1-R_{d}^{2-\alpha}\right)}{(\alpha-2)\left(R_{d}^{2}-1\right)}(2 \pi)^{\frac{\alpha}{2}} N_{\mathrm{t}}}\right)^{\frac{2}{\alpha}}\left(\prod_{m=1}^{N_{\mathrm{t}}}\left(N_{\mathrm{r}}-N_{\mathrm{t}}+m-1\right)\right)^{\frac{2}{N_{\mathrm{t} \alpha}}} \frac{1}{2^{\ln 2}},
\end{aligned}
$$

and the optimal Aloha probabilities are

$$
\begin{aligned}
& p_{\mathrm{ZF}, \mathrm{L}}^{*}=\min \left(1, \frac{\lambda_{\mathrm{ZF}, \mathrm{L}}^{*}}{\lambda}\right) \\
& p_{\mathrm{SIC}, \mathrm{L}}^{*}=\min \left(1, \frac{\lambda_{\mathrm{SIC}, \mathrm{L}}^{*}}{\lambda}\right) .
\end{aligned}
$$




\section{Conclusions}

We considered a random wireless network with multiple transmit and receive antennas and examined the benefits of using MIMO techniques for obtaining multiplexing gains from the ergodic spectral efficiency point-of-view. Assuming two different types of CSI at receivers, we gave exact analytical expressions and scaling laws for the ergodic spectral efficiency. The main finding is that the ergodic spectral efficiency increases linearly with both the density of nodes and the number of transmit streams, provided that the number of antennas scales in a particular polynomial function with the density. When local CSI with ZF-SIC detection is employed, the lower bound of the scaling law increases linearly with the density of nodes, the path-loss exponent and the number of transmit antennas provided the ratio between transmit and receive antennas scales in a linear way with the density.

There are many interesting directions left as future work. One possible direction is to consider antenna correlation effects in both transmit and receive antennas, and to analyze how the correlation effects change the scaling laws. Assuming a MIMO random network with finite feedback, it would also be interesting to investigate the benefits of a closed-loop MIMO transmission technique over the open-loop transmission method examined here. Another direction is to assume a MIMO heterogeneous network and to investigate the optimum number of data streams as a function of the density of nodes.

\section{APPENDIX A}

\section{A LEMMA FOR CAPACITY ANALYSIS}

The following lemma presented in [35] will be useful below.

Lemma 1: Let $x_{1}, \ldots, x_{N}, y_{1}, \ldots, y_{M}$ be arbitrary non-negative random variables. Then

$$
\mathbb{E}\left[\ln \left(1+\frac{\sum_{n=1}^{N} x_{n}}{\sum_{m=1}^{M} y_{m}+1}\right)\right]=\int_{0}^{\infty} \frac{\mathcal{M}_{y}(z)-\mathcal{M}_{x, y}(z)}{z} \exp (-z) d z,
$$

where $\mathcal{M}_{y}(z)=\mathbb{E}\left[e^{-z \sum_{m=1}^{M} y_{m}}\right]$ and $\mathbb{M}_{x, y}(z)=\mathbb{E}\left[e^{-z\left(\sum_{n=1}^{N} x_{n}+\sum_{m=1}^{M} y_{m}\right)}\right]$.

Proof: See [35].

The following lemma, proved in [33, Appendix B], will also be used: 
Lemma 2: Let $X>0$ and $Y>0$ be independent non-negative random variables such that $\mathbb{E}[X]<\infty, \mathbb{E}[Y]<\infty$, and $\mathbb{E}\left[\frac{1}{Y}\right]<\infty$. Then,

$$
\log _{2}\left(1+\frac{\exp (\mathbb{E}[\ln (X)])}{\mathbb{E}[Y]}\right) \leq \mathbb{E}_{X, Y}\left[\log _{2}\left(1+\frac{X}{Y}\right)\right] \leq \log _{2}\left(1+\mathbb{E}[X] \mathbb{E}\left[\frac{1}{Y}\right]\right)
$$

\section{APPENDIX B}

\section{PROOF OF THEOREM 1 AND 2}

Let $X$ and $Y$ be two independent non-negative random variables with $a \in \mathbb{R}^{+}$, Lemma 1 becomes

$$
\mathbb{E}\left[\ln \left(1+\frac{X}{Y+a}\right)\right]=\int_{0}^{\infty} \frac{e^{-a z}}{z}\left(1-\mathbb{E}\left[e^{-z X}\right]\right) \mathbb{E}\left[e^{-z Y}\right] d z .
$$

We first prove Theorem 11. Given $d_{0,0}=d$ for the typical link and $N_{\mathrm{t}, k}=t$, applying (51), the ergodic spectral efficiency for the $m$-th data stream of the typical link is

$$
\begin{aligned}
& \mathbb{E}\left[\log _{2}\left(1+\frac{H_{0,0}(m)}{d_{0,0}^{\alpha} I_{0}(m)+\frac{d_{0,0}^{\alpha} t \sigma^{2}}{P}}\right) \mid d_{0,0}=d, N_{\mathrm{t} 0}=t\right] \\
= & \frac{1}{\ln 2} \int_{0}^{\infty} \frac{e^{-\frac{d^{\alpha} t \sigma^{2}}{P} z}}{z}\left(1-\mathbb{E}\left[e^{-z H_{0,0}(m)}\right]\right) \mathbb{E}\left[e^{-z d^{\alpha} I_{0}(m)}\right] d z .
\end{aligned}
$$

Let us define $I_{0}(m)=\bar{I}_{01}(m)+\bar{I}_{02}(m)+\ldots+\bar{I}_{0 N_{\mathrm{r}}}(m)$, where $\bar{I}_{0 k}(m)$ is the interference from nodes which have $k$-transmit antennas. Then, the Laplace transform of the interference $I_{0}(m)$ is

$$
\mathcal{L}_{I_{0}(m)}=\mathbb{E}\left[e^{-s I_{0}(m)}\right]=\mathbb{E}\left[e^{-s \sum_{k=1}^{N_{\mathrm{r}}} \bar{I}_{0 k}(m)}\right]=\prod_{k=1}^{N_{\mathrm{r}}} \mathbb{E}\left[e^{-s \bar{I}_{0 k}(m)}\right]=\prod_{k=1}^{N_{\mathrm{r}}} \mathcal{L}_{\bar{I}_{0 k}(m)}(s) .
$$

The Laplace transform of $\bar{I}_{0 i}(m)$ is

$$
\begin{aligned}
\mathcal{L}_{\bar{I}_{0 k}(m)}(s) & \stackrel{(a)}{=} \exp \left(-\int_{\mathbb{R}^{2}} \mathbb{E}_{p}\left[1-e^{-s \frac{p}{r^{\alpha}}}\right] \lambda p_{k} d x d y\right) \\
& \stackrel{(b)}{=} \exp \left(-\lambda p_{k} \int_{0}^{2 \pi} \int_{0}^{\infty} \mathbb{E}_{p}\left[1-e^{-s \frac{p}{r^{\alpha}}}\right] r d r d \theta\right) \\
& \stackrel{(c)}{=} \exp \left(-2 \pi \lambda p_{k} \mathbb{E}_{p}\left[\int_{0}^{\infty}\left(1-e^{-s \frac{p}{r^{\alpha}}}\right) r d r\right]\right) \\
& \stackrel{(d)}{=} \exp \left(-\pi \lambda p_{k} \mathbb{E}_{p}\left[(s p)^{\frac{2}{\alpha}} \int_{0}^{\infty}\left(1-e^{-u}\right) \frac{-2}{\alpha} \frac{1}{u^{1+\frac{2}{\alpha}}} d u\right]\right) \\
& \stackrel{(e)}{=} \exp \left(-\pi \lambda p_{k} \mathbb{E}_{p}\left[(s p)^{\frac{2}{\alpha}} \int_{0}^{\infty} e^{-u} u^{-\frac{2}{\alpha}} d u\right]\right) \\
& \stackrel{(f)}{=} \exp \left(-\pi \lambda p_{k} \Gamma\left(1-\frac{2}{\alpha}\right) \mathbb{E}_{p}\left[(s p)^{\frac{2}{\alpha}}\right]\right) \\
& \stackrel{(g)}{=} \exp \left(-\pi \lambda p_{k} s^{\frac{2}{\alpha}} \Gamma\left(1-\frac{2}{\alpha}\right) \frac{\Gamma\left(k+\frac{2}{\alpha}\right)}{\Gamma(k)}\right) .
\end{aligned}
$$


(a) comes from the thinning, the displacement theorem, and the independent marking of PPP [17]; $p$ is the inter-node interference power when $\mathbf{v}_{0}(m)$ is applied. (b) is obtained by changing from Cartesian coordinates to polar coordinates; (c) is by Fubini's theorem. (d) follows from the change of variable $u=\frac{s p}{r^{\alpha}}$; (e) comes from the integration by part; (f) is by the definition of the Gamma function and $(\mathrm{g})$ comes from the fact that $p$ is a chi-squared random variable with $2 k$ degrees of freedom.

So, the Laplace transform of the interference $I_{0}(m)$ at $z d^{\alpha}$ is

$$
\mathbb{E}\left[e^{-z d^{\alpha} I_{0}(m)}\right]=\prod_{k=1}^{N_{\mathrm{r}}} \exp \left(-\pi \lambda p_{k} d^{2} z^{\frac{2}{\alpha}} \frac{\Gamma\left(k+\frac{2}{\alpha}\right)}{\Gamma(k)} \Gamma\left(1-\frac{2}{\alpha}\right)\right),
$$

which comes from the independent thinning and the superposition of PPP with probability generating functional (PGFL) of PPP [17]. By plugging (54) into (52), we obtain

$$
\begin{aligned}
& \frac{1}{\ln 2} \int_{0}^{\infty} \frac{e^{-\frac{d^{\alpha} \sigma_{\sigma} \sigma^{2}}{P}}}{z}\left(1-\mathbb{E}\left[e^{-z H_{0,0}(m)}\right]\right) \exp \left(-\pi \lambda d^{2} z^{\frac{2}{\alpha}} \sum_{k=1}^{N_{\mathrm{r}}} p_{k} \frac{\Gamma\left(k+\frac{2}{\alpha}\right)}{\Gamma(k)} \Gamma\left(1-\frac{2}{\alpha}\right)\right) d z \\
\stackrel{(a)}{=} & \frac{\alpha}{2 \ln 2} \int_{0}^{\infty} \frac{e^{-\frac{d^{\alpha} t \sigma^{2}}{P}\left(\lambda \pi d^{2} \sum_{k=1}^{N_{\mathrm{r}}} p_{k} \frac{\Gamma\left(k+\frac{2}{\alpha}\right) \Gamma\left(1-\frac{2}{\alpha}\right)}{\Gamma(k) u}\right)^{-\frac{\alpha}{2}}}}{u} \\
& \times\left(1-\mathbb{E}\left[e^{-\left(\lambda \pi d^{2} \sum_{k=1}^{N_{\mathrm{r}}} p_{k} \frac{\Gamma\left(k+\frac{2}{\alpha}\right) \Gamma\left(1-\frac{2}{\alpha}\right)}{\Gamma(k) u}\right)^{-\frac{\alpha}{2}} H_{k, k}(m)}\right]\right) e^{-u} d u \\
\stackrel{(b)}{=} & \left.\frac{\alpha}{2 \ln 2} \int_{0}^{\infty} \frac{e^{-\frac{d^{\alpha} t \sigma^{2}}{P}\left(\lambda \pi d^{2} \sum_{k=1}^{N_{\mathrm{r}}} p_{k} \frac{\Gamma\left(k+\frac{2}{\Gamma}\right) \Gamma\left(1-\frac{2}{\alpha}\right)}{\Gamma(k) u}\right)^{-\frac{\alpha}{2}}-u}}{u}\right) d u, \\
& \times\left(1-\left(\frac{1}{1+\left(\lambda \pi d^{2} \sum_{k=1}^{N_{\mathrm{r}}} p_{k} \frac{\Gamma\left(k+\frac{2}{\alpha}\right) \Gamma\left(1-\frac{2}{\alpha}\right)}{\Gamma(k) u}\right)^{-\frac{\alpha}{2}}}\right)\right.
\end{aligned}
$$

where (a) comes from a variable change, and (b) follows from deconditioning $H_{k, k}(m)$ which is a Chi-squared random variable with $2\left(N_{\mathrm{r}}-t+1\right)$ degrees of freedom. Since $Y_{k}$ is uniformly distributed in the ring centered at $X_{k}$, we obtain (15) by considering all data streams and deconditioning w.r.t. the number of transmit antennas of the typical link.

For the ZF-SIC detection method, the main difference in the proof is that $\tilde{H}_{0,0}(m)$ is distributed 
as a Chi-squared with $2\left(N_{\mathrm{r}}-t+m\right)$ degrees of freedom, and (55) is changed to

$$
\begin{aligned}
& \frac{\alpha}{2 \ln 2} \int_{0}^{\infty} \frac{e^{-\frac{d^{\alpha} t \sigma^{2}}{P}\left(\lambda \pi d^{2} \sum_{k=1}^{N_{\mathrm{r}}} p_{k} \frac{\Gamma\left(k+\frac{2}{\alpha}\right) \Gamma\left(1-\frac{2}{\alpha}\right)}{\Gamma(k) u}\right)^{-\frac{\alpha}{2}}-u}}{u} \\
& \left(1-\left(\frac{1}{1+\left(\lambda \pi d^{2} \sum_{k=1}^{N_{\mathrm{r}}} p_{k} \frac{\Gamma\left(k+\frac{2}{\alpha}\right) \Gamma\left(1-\frac{2}{\alpha}\right)}{\Gamma(k) u}\right)^{-\frac{\alpha}{2}}}\right)^{N_{\mathrm{r}}-t+m}\right) d u,
\end{aligned}
$$

and we obtain (16) similarly.

\section{APPENDIX C}

\section{ProOF OF THEOREM 3 AND 4}

Proof: We start to derive the lower and upper bounds of (15). By applying Lemma 2 , the sum spectral efficiency over the network is lower bounded as follows:

$$
\begin{aligned}
\lambda \mathbb{E}^{0}\left[\sum_{m=1}^{N_{\mathrm{t}}} \log _{2}\left(1+\operatorname{SINR}_{0}^{\mathrm{ZF}}(m)\right)\right] & =\lambda \sum_{m=1}^{N_{\mathrm{t}}} \mathbb{E}_{H_{0,0}(m), d_{0,0}, I_{0}(m)}\left[\log _{2}\left(1+\frac{H_{0,0}(m) d_{0,0}^{-\alpha}}{I_{0}(m)}\right)\right] \\
& \geq \lambda \sum_{m=1}^{N_{\mathrm{t}}} \mathbb{E}_{d_{0,0}, I_{0}(m)}\left[\log _{2}\left(1+\frac{e^{\mathbb{E}\left[\ln \left(H_{0,0}(m)\right)\right]}}{d_{0,0}^{\alpha} I_{0}(m)}\right)\right] .
\end{aligned}
$$

Since $H_{0,0}(m)$ is a Chi-square random variable with $2\left(N_{\mathrm{r}}-N_{\mathrm{t}}+1\right)$ degrees of freedom,

$$
\mathbb{E}\left[\ln \left(H_{0,0}(m)\right)\right]=\psi\left(N_{\mathrm{r}}-N_{\mathrm{t}}+1\right),
$$

where

$$
\psi(n)=-\gamma+\sum_{j=1}^{n-1} \frac{1}{j},
$$

with $\gamma \simeq 0.577$, Euler's constant. By [36, Theorem 3.1],

$$
e^{\psi(x)}>x-1,
$$

and we obtain

$$
e^{\mathbb{E}\left[\ln \left(H_{0,0}(m)\right)\right]}>N_{\mathrm{r}}-N_{\mathrm{t}}+\epsilon,
$$


where $\epsilon$ is some positive number 8 . Thus, the lower bound of the sum spectral efficiency per unit area is

$$
\begin{aligned}
& \lambda \sum_{m=1}^{N_{\mathrm{t}}} \mathbb{E}_{d_{0,0}, I_{0}(m)}\left[\log _{2}\left(1+\frac{N_{\mathrm{r}}-N_{\mathrm{t}}+\epsilon}{d_{0,0}^{\alpha} I_{0}(m)}\right)\right] \\
& \stackrel{(a)}{=} \frac{\lambda}{\ln 2} \sum_{m=1}^{N_{\mathrm{t}}} \int_{0}^{\infty} \frac{1}{z}\left(1-e^{-z\left(N_{\mathrm{r}}-N_{\mathrm{t}}+\epsilon\right)}\right) \mathbb{E}_{d_{0,0}, I_{0}(m)}\left[e^{-z d_{0,0}^{\alpha} I_{0}(m)}\right] d z \\
& \stackrel{(b)}{=} \frac{\lambda}{\ln 2} \sum_{m=1}^{N_{\mathrm{t}}} \int_{0}^{\infty} \frac{1}{z}\left(1-e^{-z\left(N_{\mathrm{r}}-N_{\mathrm{t}}+\epsilon\right)}\right) \mathbb{E}_{d_{0,0}}\left[\exp \left(-\lambda \pi d_{0,0}^{2} z^{\frac{2}{\alpha}} \frac{\Gamma\left(N_{\mathrm{t}}+\frac{2}{\alpha}\right) \Gamma\left(1-\frac{2}{\alpha}\right)}{\Gamma\left(N_{\mathrm{t}}\right)}\right)\right] d z \\
& \stackrel{(c)}{\geq} \frac{\lambda}{\ln 2} \sum_{m=1}^{N_{\mathrm{t}}} \int_{0}^{\infty} \frac{1}{z}\left(1-e^{-z\left(N_{\mathrm{r}}-N_{\mathrm{t}}+\epsilon\right)}\right) \exp \left(-\lambda \pi \mathbb{E}\left[d_{0,0}^{2}\right] z^{\frac{2}{\alpha}} \frac{\Gamma\left(N_{\mathrm{t}}+\frac{2}{\alpha}\right) \Gamma\left(1-\frac{2}{\alpha}\right)}{\Gamma\left(N_{\mathrm{t}}\right)}\right) d z \\
& \stackrel{(d)}{=} \frac{\lambda \alpha}{2 \ln 2} \sum_{m=1}^{N_{\mathrm{t}}} \int_{0}^{\infty} \frac{1}{u} e^{-u}\left(1-e^{-\left(\frac{2 \Gamma\left(N_{\mathrm{t}}\right)}{\Gamma\left(N_{\mathrm{t}}+\frac{2}{\alpha}\right) \Gamma\left(1-\frac{2}{\alpha}\right)}\right)^{\frac{\alpha}{2}} \frac{N_{\mathrm{r}}-N_{\mathrm{t}}+\epsilon}{\left(\lambda \pi\left(R_{d}^{2}+1\right)\right)^{\frac{\alpha}{2}}} u^{\frac{\alpha}{2}}}\right) d u \\
& \stackrel{(e)}{\geq} \frac{\lambda}{\ln 2} \sum_{m=1}^{N_{\mathrm{t}}} \int_{0}^{\infty} \frac{1}{u} e^{-u^{\frac{\alpha}{2}}}\left(1-e^{-\left(\frac{2 \Gamma\left(N_{\mathrm{t}}\right)}{\Gamma\left(N_{\mathrm{t}}+\frac{2}{\alpha}\right) \Gamma\left(1-\frac{2}{\alpha}\right)}\right)^{\frac{\alpha}{2}} \frac{N_{\mathrm{r}}-N_{\mathrm{t}}+\epsilon}{\left(\lambda \pi\left(R_{d}^{2}+1\right)\right)^{\frac{\alpha}{2}}} u^{\frac{\alpha}{2}}}\right) d u \\
& \stackrel{(f)}{=} \frac{2 \lambda N_{\mathrm{t}}}{\alpha} \log _{2}\left(1+\left(\frac{2 \Gamma\left(N_{\mathrm{t}}\right)}{\Gamma\left(N_{\mathrm{t}}+\frac{2}{\alpha}\right) \Gamma\left(1-\frac{2}{\alpha}\right)}\right)^{\frac{\alpha}{2}} \frac{N_{\mathrm{r}}-N_{\mathrm{t}}+\epsilon}{\left(\lambda \pi\left(R_{d}^{2}+1\right)\right)^{\frac{\alpha}{2}}}\right) \\
& \stackrel{(g)}{\geq} \frac{2 \lambda N_{\mathrm{t}}}{\alpha} \log _{2}\left(1+\frac{1}{N_{\mathrm{t}}}\left(\frac{2}{\Gamma\left(1-\frac{2}{\alpha}\right)}\right)^{\frac{\alpha}{2}} \frac{N_{\mathrm{r}}-N_{\mathrm{t}}+\epsilon}{\left(\lambda \pi\left(R_{d}^{2}+1\right)\right)^{\frac{\alpha}{2}}}\right),
\end{aligned}
$$

where (a) follows from Lemma 1, (b) comes from the expression for the interference of the Laplace functional of PPP, (c) follows from Lemma 2, (d) comes from a variable change and the fact that $\mathbb{E}\left[d_{k, k}^{2}\right]=\frac{R_{d}^{2}+1}{2}$, (e) comes from the fact that $e^{-u} \geq \frac{2}{\alpha} e^{-u^{\frac{\alpha}{2}}}$ when $u \geq 0$ and $\alpha>2$, (f) is obtained by $\int_{0}^{\infty} \frac{1}{u} e^{-u^{\frac{\alpha}{2}}}\left(1-e^{-b \times u^{\frac{\alpha}{2}}}\right) d u=\frac{2}{\alpha} \log (1+b)$, and (g) comes from

$$
\frac{\Gamma\left(N_{\mathrm{t}}\right)}{\Gamma\left(N_{\mathrm{t}}+\frac{2}{\alpha}\right)} \geq N_{\mathrm{t}}^{-\frac{2}{\alpha}}
$$

Using the assumption that $N_{\mathrm{t}}=c_{1} \lambda^{\beta_{1}}$ and $N_{\mathrm{r}}=c_{2} \lambda^{\beta_{2}}$, we obtain

$$
\lim _{\lambda \rightarrow \infty} C^{\mathrm{ZF}}=\Omega\left(\lambda^{\beta_{1}+1} \log _{2}\left(1+\lambda^{\beta_{2}-\beta_{1}-\frac{\alpha}{2}}\right)\right)
$$

as $\lambda$ goes to infinity.

\footnotetext{
${ }^{8}$ With a numerical approach, the gap of $e^{\psi(x)}$ and $x-1$ is lower bounded by 0.4 . For obtaining lower bound of the sum spectral efficiency (and scaling law of it), we just put $\epsilon$ to prevent the lower bound becoming 0 .
} 
Next, we derive an upper bound when the receiver applies ZF. In the interference limited regime,

$$
\begin{aligned}
\lambda \mathbb{E} & {\left[\sum_{m=1}^{N_{\mathrm{t}}} \log _{2}\left(1+\operatorname{SINR}_{0}^{\mathrm{ZF}}(m)\right)\right]=\lambda \sum_{m=1}^{N_{\mathrm{t}}} \mathbb{E}_{H_{0,0}(m), d_{0,0}, I_{0}(m)}\left[\log _{2}\left(1+\frac{H_{0,0}(m) d_{0,0}^{-\alpha}}{I_{0}(m)}\right)\right] } \\
& \stackrel{(a)}{\leq} \lambda N_{\mathrm{t}} \log _{2}\left(1+\mathbb{E}\left[d_{0,0}^{-\alpha}\right] \mathbb{E}\left[H_{0,0}(m)\right] \mathbb{E}\left[\frac{1}{I_{0}(m)}\right]\right) \\
& \stackrel{(b)}{=} \lambda N_{\mathrm{t}} \log _{2}\left(1+\frac{2\left(1-R_{d}^{2-\alpha}\right)}{(\alpha-2)\left(R_{d}^{2}-1\right)} \frac{1}{R_{d}^{\alpha}}\left(N_{\mathrm{r}}-N_{\mathrm{t}}+1\right) \frac{\Gamma\left(1+\frac{2}{\alpha}\right) \Gamma\left(N_{\mathrm{t}}\right)^{\frac{\alpha}{2}}}{\left(\lambda \pi \Gamma\left(N_{\mathrm{t}}+\frac{2}{\alpha}\right) \Gamma\left(1-\frac{2}{\alpha}\right)\right)^{\frac{\alpha}{2}}}\right),
\end{aligned}
$$

where (a) comes from Lemma 2 , and (b) follows from $\mathbb{E}\left[d_{0,0}^{-\alpha}\right]=\frac{2\left(1-R_{d}^{2-\alpha}\right)}{(\alpha-2)\left(R_{d}^{2}-1\right)}, \mathbb{E}\left[H_{0,0}(m)\right]=$ $N_{\mathrm{r}}-N_{\mathrm{t}}+1$, and the relation of $\mathbb{E}\left[\frac{1}{X}\right]=\mathbb{E}\left[\int_{0}^{\infty} e^{-s X} d s\right]$ for any positive random variable $X$. The negative moment of $I_{0}(m)$ is

$$
\mathbb{E}\left[\frac{1}{I_{0}(m)}\right]=\int_{0}^{\infty} \mathbb{E}\left[e^{-s I_{0}(m)}\right]=\int_{0}^{\infty} e^{-\lambda \pi \frac{\Gamma\left(N_{\mathrm{t}}+\frac{2}{\alpha}\right) \Gamma\left(1-\frac{2}{\alpha}\right)}{\Gamma\left(N_{\mathrm{t}}\right)} s^{\frac{2}{\alpha}}} d s=\frac{\Gamma\left(1+\frac{2}{\alpha}\right) \Gamma\left(N_{\mathrm{t}}\right)^{\frac{\alpha}{2}}}{\left(\lambda \pi \Gamma\left(N_{\mathrm{t}}+\frac{2}{\alpha}\right) \Gamma\left(1-\frac{2}{\alpha}\right)\right)^{\frac{\alpha}{2}}} .
$$

Therefore, the upper bound on the sum spectral efficiency per unit area is

$$
\begin{aligned}
C^{\mathrm{ZF}} & =\lambda N_{\mathrm{t}} \log _{2}\left(1+\frac{2\left(1-R_{d}^{2-\alpha}\right)}{(\alpha-2)\left(R_{d}^{2}-1\right)}\left(N_{\mathrm{r}}-N_{\mathrm{t}}+1\right) \frac{\Gamma\left(1+\frac{2}{\alpha}\right) \Gamma\left(N_{\mathrm{t}}\right)^{\frac{\alpha}{2}}}{\left(\lambda \pi \Gamma\left(N_{\mathrm{t}}+\frac{2}{\alpha}\right) \Gamma\left(1-\frac{2}{\alpha}\right)\right)^{\frac{\alpha}{2}}}\right) \\
& \leq \lambda N_{\mathrm{t}} \log _{2}\left(1+\frac{2\left(1-R_{d}^{2-\alpha}\right)}{(\alpha-2)\left(R_{d}^{2}-1\right)}\left(N_{\mathrm{r}}-N_{\mathrm{t}}+1\right) \frac{\Gamma\left(1+\frac{2}{\alpha}\right)}{\left(\lambda \pi \Gamma\left(1-\frac{2}{\alpha}\right)\right)^{\frac{\alpha}{2}}}\left(\left(N_{\mathrm{t}}-1\right)^{-\frac{2}{\alpha}}\right)^{\frac{\alpha}{2}}\right),
\end{aligned}
$$

where the last inequality comes from

$$
\frac{\Gamma(x)}{\Gamma\left(x+\frac{2}{\alpha}\right)} \leq(x-1)^{-\frac{2}{\alpha}}
$$

By letting $\lambda$ tend to infinity, we obtain

$$
\lim _{\lambda \rightarrow \infty} C^{\mathrm{ZF}}=\mathcal{O}\left(\lambda^{\beta_{1}+1} \log _{2}\left(1+\lambda^{\beta_{2}-\beta_{1}-\frac{\alpha}{2}}\right)\right) .
$$

Equations (64) and (69) conclude the proof of Theorem 3 .

The proof of Theorem 4 is analogous to that of Theorem 3 . The main difference consists in changing $H_{0,0}(m) \sim \chi_{2\left(N_{\mathrm{r}}-N_{\mathrm{t}}+1\right)}^{2}$ to $\tilde{H}_{0,0}(m) \sim \chi_{2\left(N_{\mathrm{r}}-N_{\mathrm{t}}+m\right)}^{2}$. The lower bound of the sum 
spectral efficiency per unit area becomes

$$
\begin{aligned}
& \lambda \mathbb{E}\left[\sum_{m=1}^{N_{\mathrm{t}}} \log _{2}\left(1+\operatorname{SINR}_{0}^{\mathrm{SIC}}(m)\right)\right] \\
& >\frac{2 \lambda}{\alpha} \sum_{m=1}^{N_{\mathrm{t}}} \log _{2}\left(1+\left(\frac{2 \Gamma\left(N_{\mathrm{t}}\right)}{\Gamma\left(N_{\mathrm{t}}+\frac{2}{\alpha}\right) \Gamma\left(1-\frac{2}{\alpha}\right)}\right)^{\frac{\alpha}{2}} \frac{N_{\mathrm{r}}-N_{\mathrm{t}}+m-1+\epsilon}{\left(\lambda \pi\left(R_{d}^{2}+1\right)\right)^{\frac{\alpha}{2}}}\right) \\
& >\frac{2 \lambda N_{\mathrm{t}}}{\alpha} \log _{2}\left(1+\left(\frac{2}{\pi\left(R_{d}^{2}+1\right) \Gamma\left(1-\frac{2}{\alpha}\right)}\right)^{\frac{\alpha}{2}} \frac{N_{\mathrm{r}}-N_{\mathrm{t}}+\epsilon}{N_{\mathrm{t}}} \lambda^{-\frac{\alpha}{2}}\right)
\end{aligned}
$$

and the upper bound becomes

$$
\begin{aligned}
& \lambda \mathbb{E}\left[\sum_{m=1}^{N_{\mathrm{t}}} \log _{2}\left(1+\operatorname{SINR}_{0}^{\mathrm{SIC}}(m)\right)\right] \\
& \leq \lambda \sum_{m=1}^{N_{\mathrm{t}}} \log _{2}\left(1+\frac{2\left(1-R_{d}^{2-\alpha}\right)}{(\alpha-2)\left(R_{d}^{2}-1\right)}\left(N_{\mathrm{r}}-N_{\mathrm{t}}+m\right) \frac{\Gamma\left(1+\frac{2}{\alpha}\right) \Gamma\left(N_{\mathrm{t}}\right)^{\frac{\alpha}{2}}}{\left(\lambda \pi \Gamma\left(N_{\mathrm{t}}+\frac{2}{\alpha}\right) \Gamma\left(1-\frac{2}{\alpha}\right)\right)^{\frac{\alpha}{2}}}\right) \\
& <\lambda N_{\mathrm{t}} \log _{2}\left(1+\frac{2\left(1-R_{d}^{2-\alpha}\right)}{(\alpha-2)\left(R_{d}^{2}-1\right)} \frac{\Gamma\left(1+\frac{2}{\alpha}\right)}{\left(\pi \Gamma\left(1-\frac{2}{\alpha}\right)\right)^{\frac{\alpha}{2}}} N_{\mathrm{r}}\left(\frac{\Gamma\left(N_{\mathrm{t}}\right)}{\Gamma\left(N_{\mathrm{t}}+\frac{2}{\alpha}\right)}\right)^{\frac{\alpha}{2}} \lambda^{-\frac{\alpha}{2}}\right) \\
& \leq \lambda N_{\mathrm{t}} \log _{2}\left(1+\frac{2\left(1-R_{d}^{2-\alpha}\right)}{(\alpha-2)\left(R_{d}^{2}-1\right)} \frac{\Gamma\left(1+\frac{2}{\alpha}\right)}{\left(\pi \Gamma\left(1-\frac{2}{\alpha}\right)\right)^{\frac{\alpha}{2}}} \frac{N_{\mathrm{r}}}{N_{\mathrm{t}}-1} \lambda^{-\frac{\alpha}{2}}\right),
\end{aligned}
$$

where the last inequality comes from (68). With the foregoing assumptions, the scaling law of the sum spectral per unit area with respect to the density becomes $\Theta\left(\lambda^{\beta_{1}+1} \log _{2}\left(1+\lambda^{\beta_{2}-\beta_{1}-\frac{\alpha}{2}}\right)\right)$.

\section{APPENDIX D}

\section{PROOF OF THEOREM 5 AND 6}

We use Lemma 1 again. We start to derive the ZF-receiver case. Conditioned on $d_{k, k}=d$, the spectral efficiency of the $m$-th data stream of the typical link is

$$
\begin{aligned}
\mathbb{E}\left[\log _{2}\left(1+\frac{\tilde{H}_{0,0}(m) d_{0,0}^{-\alpha}}{\tilde{I}_{0}(m)+\frac{N_{t} \sigma^{2}}{P}}\right) \mid d_{0,0}=d\right] & =\frac{1}{\ln 2} \int_{0}^{\infty} \frac{e^{-\frac{N_{t} \sigma^{2}}{P} z}}{z}\left(1-\mathbb{E}\left[e^{-z H_{0,0}(m)} d^{-\alpha}\right]\right) \\
& \times \mathbb{E}\left[e^{-z \sum_{j=L+1}^{\infty} \tilde{H}_{0,0_{j}}(m) d_{0,0_{j}}^{-\alpha}}\right] d z
\end{aligned}
$$

by Lemma 11 Since $\tilde{H}_{0,0}(m)$ is Chi-square distributed with $2\left(N_{\mathrm{r}}-(L+1) N_{\mathrm{t}}+1\right)$ distributed,

$$
\mathbb{E}\left[e^{-z \tilde{H}_{0,0}(m) d^{-\alpha}}\right]=\frac{1}{\left(1+z d^{-\alpha}\right)^{N_{\mathrm{r}}-(L+1) N_{\mathrm{t}}+1}} .
$$


The Laplace transform of $\tilde{I}_{0}(m)$ for the given $L$ is

$$
\mathcal{L}_{\tilde{I}_{0}(m)}(L ; s)=\mathbb{E}\left[e^{-z \sum_{j=L+1}^{\infty} \tilde{H}_{0,0_{j}}(m) d_{0,0_{j}}^{-\alpha}}\right] .
$$

Under the condition that the $L$-th nearest interferer's distance is $r$, the Laplace transform is obtained as

$$
\begin{aligned}
\mathcal{L}_{\tilde{I}_{0} \mid d_{0, L}=r}(L ; s) & =\mathbb{E}\left[e^{\left.-z \sum_{j=L+1}^{\infty} \tilde{H}_{0,0_{j}}(m) d_{0,0_{j}}^{-\alpha} \mid\left\{d_{0, L}=r\right\}\right]}\right. \\
& \stackrel{(a)}{=} \mathbb{E}\left[\prod_{d_{0,0_{j}} \in \Phi \backslash \mathcal{B}(0, r)} \frac{1}{\left(1+z d_{0,0_{j}}^{-\alpha}\right)^{N_{\mathrm{t}}}} \mid\left\{d_{0, L}=r\right\}\right] \\
& \stackrel{(b)}{=} \exp \left(-\pi \lambda \int_{u=r^{2}}^{\infty} 1-\frac{1}{\left(1+z u^{-\frac{\alpha}{2}}\right)^{N_{\mathrm{t}}}} d u\right),
\end{aligned}
$$

where (a) comes from the fact that $\tilde{H}_{0,0_{j}}(m) \sim \chi_{2 N_{\mathrm{t}}}^{2}$ and (b) follows from PGFL. The distribution of $r$ is given in [37] and by unconditioning with respect to it,

$$
\mathcal{L}_{\tilde{I}_{0}}(L ; s)=\int_{0}^{\infty} \exp \left(-\pi \lambda \int_{u=r^{2}}^{\infty} 1-\frac{1}{\left(1+z u^{-\frac{\alpha}{2}}\right)^{N_{\mathrm{t}}}} d u\right) \frac{2\left(\lambda \pi r^{2}\right)^{L}}{r \Gamma(L)} e^{-\lambda \pi r^{2}} d r .
$$

Thus, the sum spectral efficiency conditioned on $d_{k, k}=d$ can be written as

$$
\begin{aligned}
& \mathbb{E}\left[\log _{2}\left(1+\frac{\tilde{H}_{0,0}(m) d^{-\alpha}}{\tilde{I}_{0}(m)+\frac{\sigma^{2} N_{\mathrm{t}}}{P}}\right) \mid\left\{d_{0,0}=d\right\}\right] \\
& =\frac{1}{\ln 2} \int_{0}^{\infty} \frac{e^{-\frac{s N_{\mathrm{t}} \sigma^{2}}{P}}}{s}\left[1-\frac{1}{\left(1+z d^{-\alpha}\right)^{N_{\mathrm{r}}-(L+1) N_{\mathrm{t}}+1}}\right] \mathcal{L}_{\tilde{I}_{0}}(L ; s) d s .
\end{aligned}
$$

We obtain the announced result when using the fact that $d_{0,0}$ is uniformly distributed in a ring with radii $\left(1, R_{d}\right)$.

The result for ZF-SIC follows by the same arguments, using the fact that $\tilde{H}_{0,0}(m)$ is Chi-square random variable with $2\left(N_{\mathrm{r}}-N_{\mathrm{t}}+m\right)$ degrees of freedom. 


\section{APPENDIX E}

\section{PROOF OF THEOREM 7 AND 8}

Proof: We start the proof of Theorem 7 , The lower bound of (36) is

$$
\begin{aligned}
\lambda \mathbb{E}\left[\sum_{m=1}^{N_{\mathrm{t}}} \log _{2}\left(1+\operatorname{SINR}_{0, L}^{\mathrm{ZF}}(m)\right)\right] & =\lambda \sum_{m=1}^{N_{\mathrm{t}}} \mathbb{E}_{\tilde{H}_{0,0} d_{0,0}, \tilde{I}_{0}(m)}\left[\log _{2}\left(1+\frac{\tilde{H}_{0,0}(m) d_{0,0}^{-\alpha}}{\tilde{I}_{0}(m)}\right)\right] \\
& \geq \lambda \sum_{m=1}^{N_{\mathrm{t}}} \log _{2}\left(1+\frac{e^{\mathbb{E}\left[\ln \left(\tilde{H}_{0,0}(m)\right)\right]}}{\mathbb{E}\left[d_{0,0}^{-\alpha}\right] \mathbb{E}\left[\tilde{I}_{0}(m)\right]}\right) \\
& \stackrel{(b)}{>} \lambda \sum_{m=1}^{N_{\mathrm{t}}} \log _{2}\left(1+\frac{N_{\mathrm{r}}-(L+1) N_{\mathrm{t}}+\epsilon}{\frac{2\left(1-R_{d}^{2-\alpha}\right)}{(\alpha-2)\left(R_{d}^{2}-1\right)} \mathbb{E}\left[\tilde{I}_{k}(m)\right]}\right),
\end{aligned}
$$

where $(a)$ comes from Lemma 2, and $(b)$ comes from the inequality $(60), \mathbb{E}\left[d_{0,0}^{-\alpha}\right]=\frac{2\left(1-R_{d}^{2-\alpha}\right)}{(\alpha-2)\left(R_{d}^{2}-1\right)}$. The expectation of $\tilde{I}_{0}$ conditioned on $d_{0,0_{L}}=r$ is

$$
\mathbb{E}\left[\tilde{I}_{0}(m) \mid d_{0,0_{L}}=r\right]=\frac{2 \pi \lambda N_{\mathrm{t}}}{2-\alpha} r^{2-\alpha}
$$

By unconditioning with respect to $d_{0,0_{L}}$ whose distribution is given in [37], we get

$$
\begin{aligned}
\mathbb{E}\left[\tilde{I}_{0}(m)\right] & =\frac{2 \pi \lambda N_{\mathrm{t}}}{2-\alpha} \int_{0}^{\infty} r^{2-\alpha} \frac{2\left(\lambda \pi r^{2}\right)^{L}}{r \Gamma(L)} e^{-\lambda \pi r^{2}} d r \\
& =(2 \pi \lambda)^{\frac{\alpha}{2}} N_{\mathrm{t}} \frac{\Gamma\left(1-\frac{\alpha}{2}+L\right)}{\Gamma(L)} .
\end{aligned}
$$

By leveraging

$$
\frac{\Gamma(L)}{\Gamma\left(1-\frac{\alpha}{2}+L\right)} \geq\left(L-\frac{\alpha}{2}\right)^{\frac{\alpha}{2}-1},
$$

the lower bound becomes

$$
\begin{aligned}
\lambda \mathbb{E}\left[\sum_{m=1}^{N_{\mathrm{t}}} \log _{2}\left(1+\mathrm{SINR}_{0, L}^{\mathrm{ZF}}(m)\right)\right] & >\lambda N_{\mathrm{t}} \log _{2}\left(1+\frac{N_{\mathrm{r}}-(L+1) N_{\mathrm{t}}+\epsilon}{\frac{2\left(1-R_{d}^{2-\alpha}\right)}{(\alpha-2)\left(R_{d}^{2}-1\right)}} \frac{\Gamma(L)}{(2 \pi \lambda)^{\frac{\alpha}{2}} N_{\mathrm{t}} \Gamma\left(1-\frac{\alpha}{2}+L\right)}\right) \\
& \geq \lambda N_{\mathrm{t}} \log _{2}\left(1+\frac{N_{\mathrm{r}}-(L+1) N_{\mathrm{t}}+\epsilon}{\frac{2\left(1-R_{d}^{2-\alpha}\right)}{(\alpha-2)\left(R_{d}^{2}-1\right)}(2 \pi \lambda)^{\frac{\alpha}{2}} N_{\mathrm{t}}}\left(L-\frac{\alpha}{2}\right)^{\frac{\alpha}{2}-1}\right) \cdot(83)
\end{aligned}
$$

By plugging $N_{\mathrm{t}}=c_{1} \lambda^{\beta_{1}}, N_{\mathrm{r}}=c_{2} \lambda^{\beta_{2}}$ into (83), we obtain the following scaling law:

$$
\lim _{\lambda \rightarrow \infty} C_{L}^{\mathrm{ZF}}=\Omega\left(\lambda^{\beta_{1}+1} \log _{2}\left(1+\lambda^{\left(\beta_{2}-\beta_{1}-1\right) \frac{\alpha}{2}-\beta_{2}}\right)\right)
$$

since $L=\left\lfloor\frac{N_{\mathrm{r}}}{N_{\mathrm{t}}}\right\rfloor-1$. 
The proof of Theorem 8 is almost identical to the proof of Theorem 7 . The main difference is in the distribution of $\tilde{H}_{0,0}(m)$. The lower bound becomes

$$
\lambda \mathbb{E}\left[\sum_{m=1}^{N_{\mathrm{t}}} \log _{2}\left(1+\operatorname{SINR}_{0, L}^{\mathrm{SIC}}(m)\right)\right]>\lambda \sum_{m=1}^{N_{\mathrm{t}}} \log _{2}\left(1+\frac{N_{\mathrm{r}}-N_{\mathrm{t}}+m-1+\epsilon}{\frac{2\left(1-R_{d}^{2-\alpha}\right)}{(\alpha-2)\left(R_{d}^{2}-1\right)}(2 \pi \lambda)^{\frac{\alpha}{2}} N_{\mathrm{t}}}\left(L-\frac{\alpha}{2}\right)^{\frac{\alpha}{2}-1}\right) .
$$

With the foregoing assumptions, we obtain

$$
\lim _{\lambda \rightarrow \infty} C_{L}^{\mathrm{SIC}}=\Omega\left(\lambda^{\beta_{1}+1} \log _{2}\left(1+\lambda^{\left(\beta_{2}-\beta_{1}-1\right) \frac{\alpha}{2}}\right)\right) .
$$

\section{ACKNOWLEDGEMENT}

This work is supported in part by the National Science Foundation under Grant No. NSF-CCF-1218338 and an award from the Simons Foundation (\#197982), both to the University of Texas at Austin.

\section{REFERENCES}

[1] F. Baccelli and B. Blaszczyszyn, Stochastic Geometry and Wireless Networks: Volume 2: APPLICATIONS. Now Publishers Inc, 2009, vol. 2 .

[2] P. Gupta and P. R. Kumar, "The capacity of wireless networks," IEEE Transactions on Information Theory, vol. 46, no. 2, pp. 388-404, 2000.

[3] S. Toumpis and A. J. Goldsmith, "Capacity regions for wireless ad hoc networks," IEEE Transactions on Wireless Communications, vol. 2, no. 4, pp. 736-748, 2003.

[4] H. Hartenstein and K. P. Laberteaux, "A tutorial survey on vehicular ad hoc networks," IEEE Communications Magazine, vol. 46, no. 6, pp. 164-171, 2008.

[5] K. Doppler, M. Rinne, C. Wijting, C. B. Ribeiro, and K. Hugl, "Device-to-device communication as an underlay to LTE-advanced networks," IEEE Communications Magazine, vol. 47, no. 12, pp. 42-49, 2009.

[6] G. Fodor, E. Dahlman, G. Mildh, S. Parkvall, N. Reider, G. Miklós, and Z. Turányi, "Design aspects of network assisted device-to-device communications," IEEE Communications Magazine, vol. 50, no. 3, pp. 170-177, 2012.

[7] R. S. Blum, "MIMO capacity with interference," IEEE Journal on Selected Areas in Communications, vol. 21, no. 5, pp. 793-801, 2003.

[8] B. Chen and M. J. Gans, "MIMO communications in ad hoc networks," IEEE Transactions on Signal Processing, vol. 54, no. 7, pp. 2773-2783, 2006.

[9] A. Özgür, O. Lévêque, and D. N. Tse, "Hierarchical cooperation achieves optimal capacity scaling in ad hoc networks," IEEE Transactions on Information Theory, vol. 53, no. 10, pp. 3549-3572, 2007.

[10] A. M. Tulino and S. Verdú, Random matrix theory and wireless communications. Now Publishers Inc, 2004 , vol. 1. 
[11] X. Yu, R. M. De Moraes, H. Sadjadpour, and J. Garcia-Luna-Aceves, "Capacity of MIMO mobile wireless ad hoc networks," in International Conference on Wireless Networks, Communications and Mobile Computing, 2005, vol. 2. IEEE, 2005, pp. 1053-1058.

[12] M. Franceschetti, O. Dousse, N. David, and P. Thiran, "Closing the gap in the capacity of wireless networks via percolation theory," IEEE Transactions on Information Theory, vol. 53, no. 3, pp. 1009-1018, 2007.

[13] O. Lévêque and E. Telatar, "Information theoretic upper bounds on the capacity of large extended ad-hoc wireless networks," in Proceedings of the 2004 IEEE International Symposium on Information Theory, no. LTHI-CONF-2006-010, 2004.

[14] M. Grossglauser and D. Tse, "Mobility increases the capacity of ad-hoc wireless networks," in INFOCOM 2001. Twentieth Annual Joint Conference of the IEEE Computer and Communications Societies. Proceedings. IEEE, vol. 3. IEEE, 2001, pp. 1360-1369.

[15] R. Negi and A. Rajeswaran, "Capacity of power constrained ad-hoc networks," in INFOCOM 2004. Twenty-third AnnualJoint Conference of the IEEE Computer and Communications Societies, vol. 1. IEEE, 2004.

[16] M. Franceschetti, M. D. Migliore, and P. Minero, "The capacity of wireless networks: Information-theoretic and physical limits," IEEE Transactions on Information Theory, vol. 55, no. 8, pp. 3413-3424, 2009.

[17] F. Baccelli and B. Blaszczyszyn, Stochastic Geometry and Wireless Networks: Volume 1: THEORY. Now Publishers Inc, 2009, vol. 1.

[18] D. Stoyan, W. S. Kendall, and J. Mecke, Stochastic Geometry and its Applications, 2nd ed. Chichester: Wiley, 1995.

[19] S. P. Weber, X. Yang, J. G. Andrews, and G. De Veciana, "Transmission capacity of wireless ad hoc networks with outage constraints," IEEE Transactions on Information Theory, vol. 51, no. 12, pp. 4091-4102, 2005.

[20] J. G. Andrews, S. Weber, and M. Haenggi, "Ad hoc networks: to spread or not to spread?[ad hoc and sensor networks]," IEEE Communications Magazine, vol. 45, no. 12, pp. 84-91, 2007.

[21] S. P. Weber, J. G. Andrews, X. Yang, and G. De Veciana, “Transmission capacity of wireless ad hoc networks with successive interference cancellation," IEEE Transactions on Information Theory, vol. 53, no. 8, pp. 2799-2814, 2007.

[22] J. Blomer and N. Jindal, "Transmission capacity of wireless ad hoc networks: Successive interference cancellation vs. joint detection," in IEEE International Conference on Communications, 2009. ICC'09. IEEE, 2009, pp. 1-5.

[23] X. Zhang and M. Haenggi, "The performance of successive interference cancellation in random wireless networks," IEEE Transactions on Information Theory, vol. 60, no. 10, pp. 6368-6388, 2014.

[24] A. M. Hunter, J. G. Andrews, and S. Weber, "Transmission capacity of ad hoc networks with spatial diversity," IEEE Transactions on Wireless Communications, vol. 7, no. 12, pp. 5058-5071, 2008.

[25] N. Jindal, J. G. Andrews, and S. Weber, "Multi-antenna communication in ad hoc networks: Achieving MIMO gains with SIMO transmission,” IEEE Transactions on Communications, vol. 59, no. 2, pp. 529-540, 2011.

[26] S. Akoum, M. Kountouris, M. Debbah, and R. W. Heath, "Spatial interference mitigation for multiple input multiple output ad hoc networks: MISO gains," in IEEE 2011 Conference Record of the Forty Fifth Asilomar Conference on Signals, Systems and Computers (ASILOMAR). IEEE, 2011, pp. 708-712.

[27] R. H. Louie, M. R. McKay, and I. B. Collings, "Open-loop spatial multiplexing and diversity communications in ad hoc networks," IEEE Transactions on Information Theory, vol. 57, no. 1, pp. 317-344, 2011.

[28] K. Huang, J. G. Andrews, D. Guo, R. W. Heath, and R. A. Berry, "Spatial interference cancellation for multiantenna mobile ad hoc networks," IEEE Transactions on Information Theory, vol. 58, no. 3, pp. 1660-1676, 2012.

[29] R. Vaze and R. W. Heath, "Transmission capacity of ad-hoc networks with multiple antennas using transmit stream adaptation and interference cancellation," IEEE Transactions on Information Theory, vol. 58, no. 2, pp. 780-792, 2012. 
[30] M. Kountouris and J. G. Andrews, "Transmission capacity scaling of SDMA in wireless ad hoc networks," in IEEE Information Theory Workshop, 2009. ITW 2009. IEEE, 2009, pp. 534-538.

[31] N. Lee, D. Morales-Jimenez, A. Lozano, and R. W. Heath, "Spectral efficiency of dynamic coordinated beamforming: A stochastic geometry approach," IEEE Transactions on Wireless Communications, vol. 14, no. 1, pp. 230-241, 2015.

[32] A. Lozano and N. Jindal, "Are yesterday-s information-theoretic fading models and performance metrics adequate for the analysis of today's wireless systems?” IEEE Communications Magazine, vol. 50, no. 11, pp. 210-217, 2012.

[33] N. Lee, F. Baccelli, and R. W. Heath, "Spectral efficiency scaling laws in dense random wireless networks with multiple receive antennas," IEEE Transactions on Information Theory, vol. 62, no. 3, pp. 1344-1359, 2016.

[34] D. Tse and P. Viswanath, Fundamentals of wireless communication. Cambridge university press, 2005.

[35] K. A. Hamdi, "A useful lemma for capacity analysis of fading interference channels," IEEE Transactions on Communications, vol. 58, no. 2, pp. 411-416, 2010.

[36] A. Laforgia and P. Natalini, "On some inequalities for the gamma function," Advances in Dynamical Systems and Applications, vol. 8, no. 2, pp. 261-267, 2013.

[37] M. Haenggi, "On distances in uniformly random networks," IEEE Transactions on Information Theory, vol. 51, no. 10, pp. 3584-3586, 2005. 